Article

\title{
Selectively Pseudocompact Groups without Infinite Separable Pseudocompact Subsets ${ }^{\dagger}$
}

\author{
Dmitri Shakhmatov ${ }^{1, *}$ and Víctor Hugo Yañez ${ }^{2}$ \\ 1 Division of Mathematics, Physics and Earth Sciences, Graduate School of Science and Engineering, \\ Ehime University, Matsuyama 790-8577, Japan \\ 2 Doctor's Course, Graduate School of Science and Engineering, Ehime University, Matsuyama 790-8577, \\ Japan; victor_yanez@comunidad.unam.mx \\ * Correspondence: dmitri.shakhmatov@ehime-u.ac.jp; Tel.: +81-89-927-9558 \\ + This article is dedicated to Professor Alexander V. Arhangel'skiı̆ on the occasion of his 80th birthday.
}

Received: 31 July 2018; Accepted: 5 November 2018; Published: 16 November 2018

\begin{abstract}
We give a "naive" (i.e., using no additional set-theoretic assumptions beyond ZFC, the Zermelo-Fraenkel axioms of set theory augmented by the Axiom of Choice) example of a Boolean topological group $G$ without infinite separable pseudocompact subsets having the following "selective" compactness property: For each free ultrafilter $p$ on the set $\mathbb{N}$ of natural numbers and every sequence $\left(U_{n}\right)$ of non-empty open subsets of $G$, one can choose a point $x_{n} \in U_{n}$ for all $n \in \mathbb{N}$ in such a way that the resulting sequence $\left(x_{n}\right)$ has a $p$-limit in $G$; that is, $\left\{n \in \mathbb{N}: x_{n} \in V\right\} \in p$ for every neighbourhood $V$ of $x$ in $G$. In particular, $G$ is selectively pseudocompact (strongly pseudocompact) but not selectively sequentially pseudocompact. This answers a question of Dorantes-Aldama and the first listed author. The group $G$ above is not pseudo- $\omega$-bounded either. Furthermore, we show that the free precompact Boolean group of a topological sum $\bigoplus_{i \in I} X_{i}$, where each space $X_{i}$ is either maximal or discrete, contains no infinite separable pseudocompact subsets.
\end{abstract}

Keywords: pseudocompact; strongly pseudocompact; $p$-compact; selectively sequentially pseudocompact; pseudo- $\omega$-bounded; non-trivial convergent sequence; separable; free precompact Boolean group; reflexive group; maximal space; ultrafilter space

MSC: Primary: 22A05; Secondary: 54A20, 54D30, 54H11

All topological spaces considered in this paper are assumed to be Tychonoff and all topological groups are assumed to be Hausdorff (and thus Tychonoff as well).

As usual, $\mathbb{N}$ denotes the set of natural numbers, and $\omega$ denotes the first infinite cardinal. We freely identify $\mathbb{N}$ with $\omega$. The symbol $\beta \mathbb{N}$ denotes the Stone-Čech compactification of $\mathbb{N}$. Recall that $\beta \mathbb{N} \backslash \mathbb{N}$ can be identified with the set of all free ultrafilters on $\mathbb{N}$. For sets $X$ and $Y$, the symbol $Y^{X}$ denotes the set of all functions from $X$ to $Y$.

A group, of which each element has order 2, is called a Boolean group. Every Boolean group is abelian, so $x+x=0$ holds for each element $x$ of a Boolean group. We use $\mathbb{Z}_{2}$ to denote the unique (Boolean) group with two elements.

\section{Definitions}

Let $p$ be a free ultrafilter on $\mathbb{N}$. Recall that a point $x$ of a topological space $X$ is a $p$-limit of a sequence $\left\{x_{n}: n \in \mathbb{N}\right\}$ of points of $X$ provided that $\left\{n \in \mathbb{N}: x_{n} \in V\right\} \in p$ for every neighbourhood $V$ of $x$ in $X[1]$.

The next notion is due to Angoa, Ortiz-Castillo, and Tamariz-Mascarúa [2,3]. 
Definition 1. Let $p$ be a free ultrafilter on $\mathbb{N}$. A space $X$ is strongly $p$-pseudocompact if it has the following property: For every sequence $\left\{U_{n}: n \in \mathbb{N}\right\}$ of non-empty open subsets of $X$, one can choose a point $x_{n} \in U_{n}$ for all $n \in \mathbb{N}$ in such a way that the resulting sequence $\left\{x_{n}: n \in \mathbb{N}\right\}$ has a $p$-limit in $X$.

We shall also consider a weaker property.

Definition 2. A space $X$ is selectively pseudocompact (called also strongly pseudocompact) provided that, for every sequence $\left\{U_{n}: n \in \mathbb{N}\right\}$ of non-empty open subsets of $X$, one can choose a point $x_{n} \in U_{n}$ for all $n \in \mathbb{N}$ in such a way that the resulting sequence $\left\{x_{n}: n \in \mathbb{N}\right\}$ has a $p$-limit in $X$ for some free ultrafilter $p$ on $\mathbb{N}$ (depending on the sequence $\left\{U_{n}: n \in \mathbb{N}\right\}$ in question).

This notion was introduced by García-Ferreira and Ortiz-Castillo [4] under the name "strongly pseudocompact." Dorantes-Aldama and the first listed author gave a list of equivalent descriptions of this property in ([5], Theorem 2.1) and proposed an alternative name for it, calling a space with this property "selectively pseudocompact" ([5], Definition 2.2). This terminology was later adopted in [6].

Clearly, strongly $p$-pseudocompact spaces are selectively pseudocompact (strongly pseudocompact).

The following notion is due to Dorantes-Aldama and the first listed author ([5], Definition 2.3).

Definition 3. A space $X$ is selectively sequentially pseudocompact provided that, for every sequence $\left\{U_{n}: n \in \mathbb{N}\right\}$ of non-empty open subsets of $X$, one can choose a point $x_{n} \in U_{n}$ for all $n \in \mathbb{N}$ in such a way that the resulting sequence $\left\{x_{n}: n \in \mathbb{N}\right\}$ has a convergent subsequence.

Selectively sequentially pseudocompact spaces are selectively pseudocompact (strongly pseudocompact), while the converse does not hold in general [5].

When considering the property from Definition 1 for multiple ultrafilters $p$ simultaneously, one could obtain two natural versions as follows:

Definition 4. Let $P$ be a non-empty subset of $\beta \mathbb{N} \backslash \mathbb{N}$. A space $X$ is

(i) strongly $P$-bounded provided that, for every sequence $\left\{U_{n}: n \in \mathbb{N}\right\}$ of non-empty open subsets of $X$, one can choose a point $x_{n} \in U_{n}$ for all $n \in \mathbb{N}$ in such a way that the resulting sequence $\left\{x_{n}: n \in \mathbb{N}\right\}$ has a $p$-limit in $X$ for every $p \in P$;

(ii) strongly $P$-pseudocompact provided that $X$ is strongly $p$-pseudocompact for each $p \in P$.

The notion of strong P-boundedness is due to Angoa, Ortiz-Castillo, and Tamariz-Mascarúa [2,3]. To the best of our knowledge, the notion from Item (ii) of Definition 4 appears to be new.

For every non-empty subset $P$ of $\beta \mathbb{N} \backslash \mathbb{N}$, the implication

$$
\text { strongly } P \text {-bounded } \rightarrow \text { strongly } P \text {-pseudocompact }
$$

trivially holds. It is also clear that the larger the subset $P$ of $\beta \mathbb{N} \backslash \mathbb{N}$ is, the stronger the corresponding property of strong $P$-boundedness and strong $P$-pseudocompactness is.

Remark 1. (i) A sequence in a topological space $X$ has a $p$-limit in $X$ for every $p \in \beta \mathbb{N} \backslash \mathbb{N}$ if and only if its closure in $X$ is compact [1]. Therefore, strong $(\beta \mathbb{N} \backslash \mathbb{N})$-boundedness of a space $X$ is easily seen to be equivalent to the following property: For every sequence $\left\{U_{n}: n \in \mathbb{N}\right\}$ of non-empty open subsets of $X$, there exists a compact subset $K$ of $X$ which has a non-empty intersection with each $U_{n}$. The spaces having this property are called pseudo- $\omega$-bounded in $[2,3]$.

(ii) Infinite strongly $(\beta \mathbb{N} \backslash \mathbb{N})$-bounded spaces contain infinite compact subsets. Indeed, an infinite space $X$ contains a sequence $\left\{U_{n}: n \in \mathbb{N}\right\}$ of pairwise disjoint non-empty open subsets. If $X$ is strongly $(\beta \mathbb{N} \backslash \mathbb{N})$-bounded, then the compact subspace $K$ of $X$ as in Item (i) must be infinite. 
Recall that a space $X$ is $\omega$-bounded if every countable subset of $X$ has compact closure in $X$. A space is pseudocompact if every real-valued continuous function on it is bounded.

\section{Introduction}

The diagram in Figure 1 summarizes implications between notions introduced in Section 1.

The double arrow in Figure 1 denotes the implication which holds only in the class of topological groups and fails for general topological spaces, as has been shown in [5].

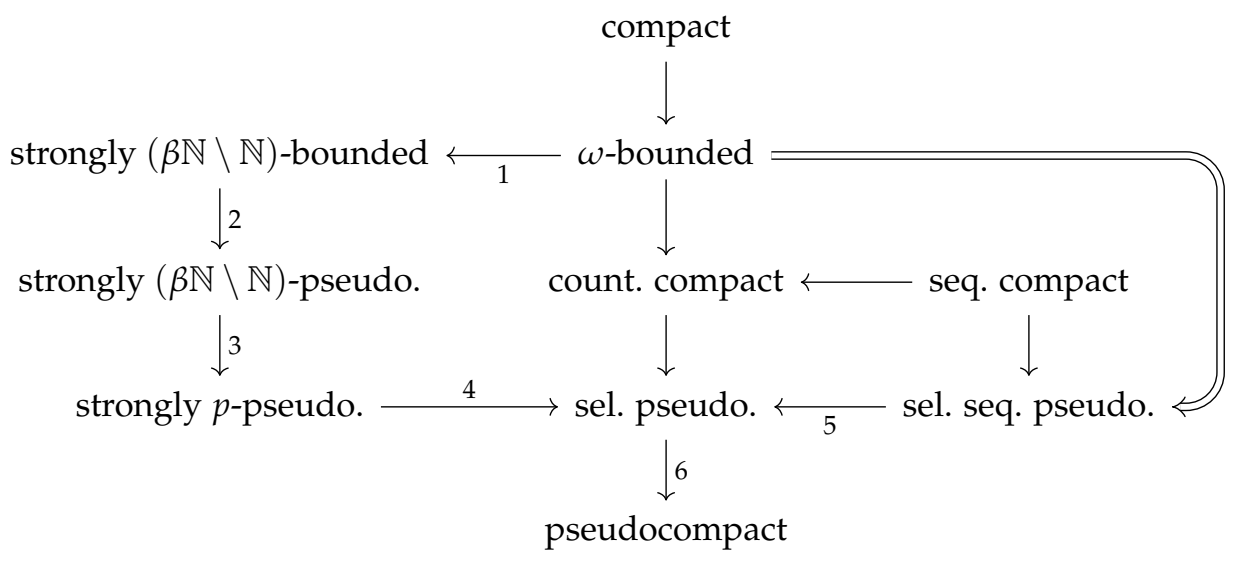

Figure 1. Implications between notions introduced in Section 1

Now we shall discuss the reversibility of arrows in Figure 1 in the class of topological groups. In Example 1, we show that Arrow 1 is not reversible. Our Corollary 2 shows that Arrow 2 is not reversible. In the text following ([7], Question 2.6), García-Ferreira and Tomita mention that there exist two free ultrafilters $p$ and $q$ on $\mathbb{N}$ and a topological group $G$ which is strongly $p$-pseudocompact but not strongly $q$-pseudocompact; in particular, $G$ is not strongly $(\beta \mathbb{N} \backslash \mathbb{N})$-pseudocompact. This shows that Arrow 3 is not reversible.

Assuming Continuum Hypothesis $\mathrm{CH}$, García-Ferreira and Tomita gave an example of a selectively pseudocompact group $G$ whose square $G^{2}$ is not selectively pseudocompact [6]. Since strong $p$-pseudocompactness is preserved by products [3] and implies selective pseudocompactness, $G$ cannot be strongly $p$-pseudocompact for any free ultrafilter $p$ on $\mathbb{N}$. This shows that Arrow 4 is not reversible under $\mathrm{CH}$. The reversibility of this arrow in ZFC alone remains unclear; see Question 6.

Next, we turn our attention to Arrows 5 and 6.

García-Ferreira and Tomita in [7] gave an example demonstrating that Arrow 6 is not reversible in the class of topological groups. The authors later showed in [8] that many examples of pseudocompact groups known in the literature fail to be selectively pseudocompact, thereby establishing relative abundance of examples witnessing non-reversibility of Arrow 6 for topological groups.

Dorantes-Aldama and the first listed author gave a consistent example of a countably compact (thus, selectively pseudocompact) topological group which is not selectively sequentially pseudocompact ([5], Example 5.7), and they asked whether such an example exists in ZFC alone ([5], Question 8.3):

Question 1. (i) Is there a ZFC example of a selectively pseudocompact (abelian) group which is not selectively sequentially pseudocompact?

(ii) Is there a ZFC example of a countably compact (abelian) group which is not selectively sequentially pseudocompact? 
We shall answer Item (i) of this question positively in Corollary 5, thereby showing that Arrow 5 of Figure 1 is not reversible in the class of topological groups. Moreover, an example we construct has much stronger property than mere selective pseudocompactness; see Corollary 4 (i).

Item (ii) of Question 1 remains open.

We refer the reader to [5] for examples witnessing the non-reversibility of arrows in Figure 1 without numbers assigned to them in the class of topological groups.

The paper is organized as follows. Section 3 contains our results related to Question 1. The main result here is Theorem 1. Corollary 2 in this section shows that the implication in Equation (1) is not reversible for $P=\beta \mathbb{N} \backslash \mathbb{N}$, even in the class of topological groups. Section 4 collects definitions of and background material on free Boolean groups over a set and free precompact Boolean groups of a topological space. In Section 5, we define a notion of a coherent map $f$ and introduce a topology on its domain so that the continuity of $f$ with respect to this topology becomes equivalent to $f$ being coherent. Splitting maps are defined in Section 6. The notion of a coherent splitting map is used in the proof of Theorem 1. The main result in this section is Theorem 2 and its Corollary 7. In Section 7, we apply the latter to show that for every infinite subset $A$ of the free precompact Boolean group $G$ of an arbitrary topological sum $\bigoplus_{k \in K} X_{k}$, where each space $X_{k}$ is either discrete or maximal, one can find a continuous group homomorphism $\varphi: G \rightarrow \mathbb{Z}_{2}$ such that the set $\{a \in A: \varphi(a)=z\}$ is infinite for every $z \in \mathbb{Z}_{2}$ (Theorem 3). This result is applied to deduce that all separable pseudocompact subsets of $G$ as above are finite (Theorem 4). In Section 8, we discuss some connections of our results to known results in the literature. Theorem 2 is proved in Section 9, and Section 10 is devoted to the proof of Theorem 1. Open questions are listed in Section 11.

\section{Results}

The main goal of the paper is to prove the following theorem.

Theorem 1. Let $\kappa$ be an infinite cardinal such that $\kappa^{\omega}=\kappa$ and $P$ be a non-empty subset of $\beta \mathbb{N} \backslash \mathbb{N}$ satisfying $|P| \leq \kappa$. There exists a dense strongly P-pseudocompact subgroup of $\mathbb{Z}_{2}^{\kappa}$ without infinite separable pseudocompact subsets.

The proof of this theorem is postponed until Section 10.

Let $\mathfrak{c}$ denote the cardinality of the continuum. Applying Theorem 1 to $P=\beta \mathbb{N} \backslash \mathbb{N}$ and $\kappa=2^{\mathfrak{c}}$, we obtain the following:

Corollary 1. There exists a dense strongly $(\beta \mathbb{N} \backslash \mathbb{N})$-pseudocompact subgroup $G$ of $\mathbb{Z}_{2}^{\mathfrak{c}}$ without infinite separable pseudocompact subsets.

The group $G$ in this corollary is clearly infinite. By Remark 1 (ii), infinite strongly $(\beta \mathbb{N} \backslash$ $\mathbb{N}$ )-bounded spaces contain infinite compact subsets (and thus, also infinite separable pseudocompact subsets). Therefore, "strong $(\beta \mathbb{N} \backslash \mathbb{N})$-pseudocompactness" of $G$ in Corollary 1 cannot be strengthened to its "strong $(\beta \mathbb{N} \backslash \mathbb{N})$-boundedness." By the same reason, the topological group G from Corollary 1 witnesses the validity of the following corollary, showing that Arrow 2 in Figure 1 is not reversible, even for topological groups.

Corollary 2. A strongly $(\beta \mathbb{N} \backslash \mathbb{N})$-pseudocompact Boolean group need not be strongly $(\beta \mathbb{N} \backslash \mathbb{N})$-bounded.

This corollary shows that the implication in Equation (1) is not reversible when $P=\beta \mathbb{N} \backslash \mathbb{N}$, even in the class of topological groups.

Given a free ultrafilter $p$ on $\mathbb{N}$, we can apply Theorem 1 to $P=\{p\}$ and $\kappa=\mathfrak{c}$ to obtain the following: 
Corollary 3. For every free ultrafilter $p$ on $\mathbb{N}$, there exists a dense strongly p-pseudocompact subgroup of $\mathbb{Z}_{2}^{\mathfrak{c}}$ without infinite separable pseudocompact subsets.

If $\kappa$ is an infinite cardinal, then every dense subset of $\mathbb{Z}_{2}^{\kappa}$ must be infinite. Since infinite selectively sequentially pseudocompact spaces contain non-trivial convergent sequences by ([5], Proposition 3.1) and convergent sequences are separable and pseudocompact, the topological groups from Theorem 1 and its Corollaries 1 and 3 are not selectively sequentially pseudocompact. In particular, we have the following corollary.

Corollary 4. (i) There exists a dense strongly $(\beta \mathbb{N} \backslash \mathbb{N})$-pseudocompact subgroup of $\mathbb{Z}_{2}^{2^{c}}$ which is not selectively sequentially pseudocompact.

(ii) For every free ultrafilter $p$ on $\mathbb{N}$, there exists a dense strongly p-pseudocompact subgroup of $\mathbb{Z}_{2}^{\mathfrak{c}}$ which is not selectively sequentially pseudocompact.

As can be seen from Figure 1, the topological groups from Corollary 4 are selectively pseudocompact. Therefore, the following particular version of Corollary 4 (ii) provides a positive answer to Question 1 (i).

Corollary 5. There exists a selectively pseudocompact Boolean group (of weight $\mathfrak{c}$ ) which is not selectively sequentially pseudocompact.

Our next remark clarifies the strength of the condition "without infinite separable pseudocompact subsets" appearing in Theorem 1 and its Corollaries 1 and 3. Indeed, this remark shows that the topological groups in these results contain no infinite subsets which belong to any of the following classes of spaces:

- countably pseudocompact;

- countably pracompact;

- countably compact;

- compact.

Remark 2. (i) Hernández and Macario [9] say that a space $X$ is countably pseudocompact if, for every countable subset $A$ of $X$, there exists a countable subset $B$ of $X$ such that $A \subseteq \bar{B}$ and $\bar{B}$ is pseudocompact. (Here $\bar{B}$ denotes the closure of $B$ in $X$.) It is immediately obvious from this definition that every infinite countably pseudocompact space contains an infinite separable pseudocompact subset.

(ii) A space $X$ is said to be countably pracompact if $X$ contains a dense set $Y$ such that every infinite subset of $Y$ has an accumulation point in $X$; see ([10], Ch. III, Sec. 4). Let $X$ be an infinite countably pracompact space, and let $Y$ be its dense subspace such that every infinite subset of $Y$ has an accumulation point in $X$. Since $X$ is infinite and $Y$ is dense in $X$, the set $Y$ must be infinite. Fix a countably infinite subset $S$ of $Y$. Then $C=\bar{S}$ is a separable space. Note that every infinite subset of $S$ has an accumulation point in C. Since $S$ is dense in $C$, it easily follows that $C$ is pseudocompact. We proved that an infinite countably pracompact space contains an infinite separable pseudocompact subset.

(iii) Since countably compact spaces are countably pracompact, it follows from (ii) that every infinite countably compact space contains an infinite separable pseudocompact subset.

(iv) Since compact spaces are countably compact, it follows from (iii) that every infinite compact space contains an infinite separable pseudocompact subset.

Remark 3. The topological groups from Theorem 1 and all its corollaries above are (Pontryagin) reflexive. Indeed, the topological group $G$ from Theorem 1 has no infinite separable pseudocompact subsets, so all compact subsets of $G$ are finite by Remark 2 (iv). Since $G$ is pseudocompact, it is reflexive by ([11], Theorem 2.8) (this also 
follows from ([12], Lemma 2.3 and Theorem 6.1)). Finally, the topological groups from all corollaries of Theorem 1 are obtained by application of this theorem, so they inherit their reflexivity from it.

Corollaries 1 and 3 and Figure 1 suggest the following natural question:

Question 2. Does there exist an infinite abelian (or even Boolean) strongly $(\beta \mathbb{N} \backslash \mathbb{N})$-bounded group $G$ satisfying one of the following conditions:

(i) $G$ is not selectively sequentially pseudocompact;

(ii) G does not have non-trivial convergent sequences?

Since infinite selectively sequentially pseudocompact spaces contain non-trivial convergent sequences by ([5], Proposition 3.1), Item (ii) of this question is stronger than Item (i). By Remark 1 (ii), Item (ii) of the question cannot be further strengthened by requiring all compact subsets of $G$ to be finite.

According to the double arrow in Figure 1, a positive answer to Question 2 (i) would provide an example of a strongly $(\beta \mathbb{N} \backslash \mathbb{N}$ )-bounded (abelian) group which is not $\omega$-bounded. However, a topological group with these properties can be easily constructed.

Example 1. Strongly $(\beta \mathbb{N} \backslash \mathbb{N})$-bounded abelian groups need not be $\omega$-bounded. Indeed, let $\kappa$ be an uncountable cardinal, and let $H$ be a countably infinite subgroup of the torus group $\mathbb{T}$. For every $h \in H$, let $c_{h} \in \mathbb{T}^{k}$ be the constant function from $\kappa$ to $\mathbb{T}$ defined by $c_{h}(\alpha)=h$ for all $\alpha \in \kappa$. Define $C=\left\{c_{h}: h \in H\right\}$. Let $D=\left\{f \in \mathbb{T}^{\kappa}:|\{\alpha \in \kappa: f(\alpha) \neq 0\}| \leq \omega\right\}$ be the $\Sigma$-product in $\mathbb{T}^{\kappa}$, and let $G$ be the smallest subgroup of $\mathbb{T}^{\kappa}$ containing $C \cup D$. Note that $C$ is a closed subgroup of $G$ which is not compact. Indeed, if $C$ were compact, its projection $H$ on a fixed coordinate would be compact as well, and as $H$ would be an infinite compact subgroup of $\mathbb{T}$, we would find that $H=\mathbb{T}$, in contradiction to our assumption that $H$ is countable. Since $C$ is a countably infinite non-compact closed subgroup of $G$, this shows that $G$ is not $\omega$-bounded. Since $G$ has a dense $\omega$-bounded subgroup $D$, it is strongly $(\beta \mathbb{N} \backslash \mathbb{N})$-bounded.

Example 1 shows that Arrow 1 in Figure 1 is not reversible, even for topological groups.

The next remark shows that the assumption in Theorem 1 that the cardinal $\kappa$ satisfies $\kappa^{\omega}=\kappa$ is essential and cannot be omitted, at least in ZFC.

Remark 4. Under the Generalized Continuum Hypothesis (GCH), if $\kappa^{\omega}>\kappa$, then every dense pseudocompact subgroup $G$ of $\mathbb{Z}_{2}^{\kappa}$ contains a non-trivial convergent sequence [13]. Further results in this direction can be found in [14].

\section{Free Boolean Groups $B(X)$ and Free Precompact Boolean Groups $F P B(X)$}

Let $X$ be a set. The set $B(X)=[X]^{<\omega}$ of all finite subsets of $X$ becomes an abelian group with the symmetric difference $E+F=(E \backslash F) \cup(F \backslash E)$ as its group operation + and the empty set as its zero element. Clearly, if $E, F \in B(X)$ are disjoint, then $E+F=E \cup F$. Each element $E$ of $B(X)$ has order 2, as $E+E=0$, so $B(X)$ is a Boolean group.

If one abuses notation by identifying an element $x \in X$ with the singleton $\{x\} \in B(X)$, then each element $E \in B(X)$ of the group $B(X)$ admits a unique decomposition $E=\sum_{x \in E} x$, so the set $X$ can be naturally considered as the set of generators of $B(X)$. (Here we agree that $\sum_{x \in \varnothing} x=0$.)

Every map $f: X \rightarrow \mathbb{Z}_{2}$ has a unique extension $\tilde{f}: B(X) \rightarrow \mathbb{Z}_{2}$ to a homomorphism of $B(X)$ to $\mathbb{Z}_{2}$ defined by

$$
\tilde{f}(E)=\sum_{x \in E} f(x) \text { for } E \in B(X),
$$

where the sum is taken in the group $\mathbb{Z}_{2}$. Since the variety $\mathcal{A}_{2}$ of all Boolean groups is generated by the single group $\mathbb{Z}_{2}$, the group $B(X)$ coincides with the free group in the variety $\mathcal{A}_{2}$ over a set $X$ [15]. 
Thus, $B(X)$ is the free Boolean group over $X$. (Note that the trivial group is the free Boolean group over the empty set.)

Recall that a topological group is precompact if it is a subgroup of some compact group, or equivalently, if its completion is compact. The class of all precompact Boolean groups forms a variety $\mathscr{V}$ of topological groups $[16,17]$. Therefore, given a topological space $X$, there exists the free object $F P B(X)$ of $X$ in $\mathscr{V}[18,19]$ which we shall call the free precompact Boolean group of $X$.

Definition 5. Let $\mathscr{V}$ be the variety of all precompact Boolean groups. For a topological space $X$, a topological group $F P B(X)$ is said to be the free precompact Boolean group of $X$ provided it satisfies two properties:

(i) $\operatorname{FPB}(X) \in \mathscr{V}$,

(ii) there exists a continuous map $\eta_{X}: X \rightarrow F P B(X)$ such that

(a) $\quad F P B(X)$ is algebraically generated by $\eta_{X}(X)$, and

(b) for every continuous map $\varphi: X \rightarrow G$ with $G \in \mathscr{V}$, there exists a continuous homomorphism $\hat{\varphi}: F P B(X) \rightarrow$ G such that $\varphi=\hat{\varphi} \circ \eta_{X}$.

A description of $F P B(X)$ as the reflection of the free (abelian) topological group of a space $X$ in the class $\mathscr{V}$ of precompact Boolean groups can be found in ([20], Section 9). Another description for zero-dimensional spaces $X$ is given in Lemma 2 below. The reason why zero-dimensionality of a space $X$ plays such an important role can be seen from the following lemma.

Lemma 1. For a topological space $X$, the following conditions are equivalent:

(i) The map $\eta_{X}$ from Item (ii) of Definition 5 is a homeomorphic embedding;

(ii) $\mathrm{X}$ is zero-dimensional.

Proof. (i) $\rightarrow$ (ii) Since $F P B(X) \in \mathscr{V}$ by Definition 5 (i), $F P B(X)$ is a precompact Boolean group. Then the completion $K$ of $F P B(X)$ is a compact Boolean group, so $K$ is zero-dimensional. Since $\eta_{X}(X) \subseteq$ $F P B(X) \subseteq K$, the subspace $\eta_{X}(X)$ of $K$ is zero-dimensional as well. Finally, since $\eta_{X}(X)$ is homeomorphic to $X$ by our assumption, it follows that $X$ is zero-dimensional.

(ii) $\rightarrow$ (i) Since $X$ is zero-dimensional, there exists a homeomorphic embedding $\varphi: X \rightarrow G$, where $G=\mathbb{Z}_{2}^{\kappa}$ for a suitable cardinal $\kappa$. Let $\eta_{X}$ and $\hat{\varphi}$ be as in Definition 5 (ii). Since $\varphi=\hat{\varphi} \circ \eta_{X}$ is an injection, so is $\eta_{X}$. Therefore, $\eta_{X}: X \rightarrow \eta_{X}(X)$ is a bijection, so it has its inverse map $\eta_{X}^{-1}$ : $\eta_{X}(X) \rightarrow X$. Similarly, since $\varphi: X \rightarrow \varphi(X)$ is a bijection, it has its inverse $\varphi^{-1}: \varphi(X) \rightarrow X$. Now, $\varphi \circ \eta_{X}^{-1}=\hat{\varphi} \circ \eta_{X} \circ \eta_{X}^{-1}=\hat{\varphi} \uparrow_{\eta_{X}(X)}$ by Definition 5 (ii) (b), so $\eta_{X}^{-1}=\varphi^{-1} \circ \hat{\varphi} \uparrow_{\eta_{X}(X)}$. Since $\varphi$ is a homeomorphic embedding, its inverse $\varphi^{-1}$ is continuous. Since $\hat{\varphi}$ is continuous as well, so is the composition $\eta_{X}^{-1}=\varphi^{-1} \circ \hat{\varphi} \uparrow_{\eta_{X}(X)}$. Since $\eta_{X}$ is continuous by Definition 5 (ii), we conclude that $\eta_{X}: X \rightarrow \eta_{X}(X)$ is a homeomorphism. We have proved that $\eta_{X}: X \rightarrow F P B(X)$ is a homeomorphic embedding.

Lemma 2. Let $X$ be a zero-dimensional topological space and let $\mathscr{F}_{X}$ be the family of all continuous maps $f: X \rightarrow \mathbb{Z}_{2}$ from $X$ to the group $\mathbb{Z}_{2}$ endowed with the discrete topology. Consider the initial topology $\mathscr{T}_{X}$ on $B(X)$ with respect to the family $\tilde{\mathscr{F}}_{X}=\left\{\tilde{f}: f \in \mathscr{F}_{X}\right\}$ of homomorphisms; that is, the family $\left\{\tilde{f}^{-1}(z): f \in \mathscr{F}_{X}, z \in \mathbb{Z}_{2}\right\}$ forms a subbase for the topology $\mathscr{T}_{X}$. Then the topological group $\left(B(X), \mathscr{T}_{X}\right)$ coincides with the free precompact Boolean group $F P B(X)$ of $X$, as witnessed by the natural inclusion map of $X$ into $B(X)$ (sending each $x \in X$ to $\{x\} \in B(X)$ ) taken as $\eta_{X}$. Furthermore, $\mathscr{T}_{X}$ induces on $X$ the original topology of $X$.

Proof. First, we check Items (i) and (ii) of Definition 5.

(i) Since $\mathscr{T}_{X}$ is the initial topology with respect to the family $\tilde{\mathscr{F}}_{X}$ consisting of homomorphisms into the compact group $\mathbb{Z}_{2}$, it is precompact. Since $B(X)$ is a Boolean group, we have $\left(B(X), \mathscr{T}_{X}\right) \in \mathscr{V}$. 
(ii) Item (a) is clear, as $\eta_{X}(X)=X$ algebraically generates $B(X)$. To check Item (b), suppose that $G \in \mathscr{V}$ and $\varphi: X \rightarrow G$ is a continuous map. It follows from $G \in \mathscr{V}$ that $G$ is a precompact Boolean group, so its completion $K$ is a compact Boolean group. The standard facts of the duality theory imply that $K$ is topologically isomorphic to the Cartesian product $\mathbb{Z}_{2}^{\tau}$ for some cardinal $\tau$. Therefore, we can identify $G$ with a subgroup of $\mathbb{Z}_{2}^{\tau}$.

Let $\alpha<\tau$ be arbitrary. Consider the projection $\pi_{\alpha}: \mathbb{Z}_{2}^{\tau} \rightarrow \mathbb{Z}_{2}$ on the $\alpha$ th coordinate. Then the composition map $\varphi_{\alpha}=\pi_{\alpha} \circ \varphi: X \rightarrow \mathbb{Z}_{2}$ is continuous, so $\varphi_{\alpha} \in \mathscr{F}_{X}$. Now $\tilde{\varphi}_{\alpha} \in \tilde{\mathscr{F}}_{X}$ by our definition of $\tilde{\mathscr{F}}_{X}$. Since the topology $\mathscr{T}_{X}$ has the family $\tilde{\mathscr{F}}_{X}$ as its subbase, it follows that the homomorphism $\tilde{\varphi}_{\alpha}:\left(B(X), \mathscr{T}_{X}\right) \rightarrow \mathbb{Z}_{2}$ is continuous.

Let $\hat{\varphi}: B(X) \rightarrow \mathbb{Z}_{2}^{\tau}$ be the continuous homomorphism defined by $\hat{\varphi}(E)=\left(\tilde{\varphi}_{\alpha}(E)\right)_{\alpha<\tau}$ for $E \in B(X)$. Note that $\hat{\varphi}(x)=\left(\tilde{\varphi}_{\alpha}(x)\right)_{\alpha<\tau}=\left(\varphi_{\alpha}(x)\right)_{\alpha<\tau}=\left(\pi_{\alpha}(\varphi(x))\right)_{\alpha<\tau}=\varphi(x)$ for $x \in X$, as each $\tilde{\varphi}_{\alpha}$ extends $\varphi_{\alpha}$. This shows that $\hat{\varphi} \Upsilon_{X}=\varphi$. Since $\varphi: X \rightarrow G$ is a homomorphism, $X$ algebraically generates $B(X)$, and $G$ is a subgroup of $\mathbb{Z}_{2}^{\tau}$, it follows that $\hat{\varphi}(B(X)) \subseteq G$. We have defined a continuous homomorphism $\hat{\varphi}:\left(B(X), \mathscr{T}_{X}\right) \rightarrow G$. Since $\eta_{X}: X \rightarrow B(X)$ is the natural inclusion map, from $\hat{\varphi}\left\lceil_{X}=\varphi\right.$ we conclude that $\hat{\varphi} \circ \eta_{X}=\varphi$.

It follows from (i) and (ii) that $\left(B(X), \mathscr{T}_{X}\right)$ coincides with the free precompact Boolean group $F P B(X)$ of $X$, as witnessed by the natural inclusion map of $X$ into $B(X)$ taken as $\eta_{X}$. Since $X$ is zero-dimensional, from Lemma 1 we conclude that $\eta_{X}$ is a homeomorphic embedding, which implies that $\mathscr{T}_{X}$ induces the original topology on $X$.

Definition 6. We shall say that a subspace $Y$ of a topological space $X$ is $\mathbb{Z}_{2}$-embedded in $X$ provided that every continuous map $g: Y \rightarrow \mathbb{Z}_{2}$ can be extended to a continuous map $f: X \rightarrow \mathbb{Z}_{2}$.

Remark 5. A clopen subset of a topological space is $\mathbb{Z}_{2}$-embedded in it.

We finish this section with the lemma which will be needed in the future proofs.

Lemma 3. Let $X$ be a zero-dimensional space.

(i) If $Y$ is a zero-dimensional space and $\varphi: Y \rightarrow X$ is a continuous injection, then the continuous homomorphism $\hat{\varphi}: F P B(Y) \rightarrow F P B(X)$ extending $\varphi$ is an injection as well.

(ii) If a closed subset $Y$ of $X$ is $\mathbb{Z}_{2}$-embedded in $X$, then $F P B(Y)$ is a closed subgroup of $F P B(X)$.

Proof. (i) It follows from Lemma 2 that, algebraically, $\hat{\varphi}: B(Y) \rightarrow B(X)$ and $\hat{\varphi}\lceil\Upsilon=\varphi$. Since $\varphi$ is an injection, so is $\hat{\varphi}$.

(ii) By Lemma 2, we can identify $F P B(X)$ and $F P B(Y)$ with $\left(B(X), \mathscr{T}_{X}\right)$ and $\left(B(Y), \mathscr{T}_{Y}\right)$, respectively. Since $B(Y) \subseteq B(X)$, it suffices to show that

(a) $\mathscr{T}_{X}$ induces the topology $\mathscr{T}_{Y}$ on $B(Y)$, and

(b) $B(Y)$ is $\mathscr{T}_{X}$-closed in $B(X)$.

In the proof below, we freely use notations from Lemma 2.

(a) Since $Y$ is a subspace of $X$, one has $\left\{f \uparrow_{Y}: f \in \mathscr{F}_{X}\right\} \subseteq \mathscr{F}_{Y}$. Since $Y$ is $\mathbb{Z}_{2}$-embedded in $X$, from Definition 6 we obtain the inverse inclusion $\mathscr{F}_{Y} \subseteq\left\{f \uparrow_{Y}: f \in \mathscr{F}_{X}\right\}$. This establishes the equality $\mathscr{F}_{Y}=\left\{f \uparrow_{Y}: f \in \mathscr{F}_{X}\right\}$, which implies (a) by definition of $\mathscr{T}_{X}$ and $\mathscr{T}_{Y}$.

(b) Suppose that $E \in B(X) \backslash B(Y)$. There then exists $x_{0} \in E \backslash Y$. Since $E$ is a finite subset of $X$ and $Y$ is $\mathscr{T}_{X}$-closed in $X$, the set $F=Y \cup\left(E \backslash\left\{x_{0}\right\}\right)$ is $\mathscr{T}_{X}$-closed in $X$ as well. Since $X$ is zero-dimensional, we can find a clopen subset $W$ of $X$ such that $F \subseteq W$ and $x_{0} \notin W$. Define the function $f: X \rightarrow \mathbb{Z}_{2}$ by $f(W) \subseteq\{0\}$ and $f(X \backslash W) \subseteq\{1\}$. Since $W$ is clopen in $X$, we have $f \in \mathscr{F}_{X}$, which implies $\tilde{f} \in \tilde{\mathscr{F}}_{X}$. 
Therefore, $O=\tilde{f}^{-1}(1) \in \mathscr{T}_{X}$ by our definition of $\mathscr{T}_{X}$. Since $E \backslash\left\{x_{0}\right\} \subseteq F \subseteq W \subseteq f^{-1}(0) \subseteq \tilde{f}^{-1}(0)$ and $x_{0} \in X \backslash W \subseteq f^{-1}(1) \subseteq \tilde{f}^{-1}(1)$, we have

$$
\tilde{f}(E)=\sum_{x \in E} \tilde{f}(x)=\tilde{f}\left(x_{0}\right)+\sum_{x \in E \backslash\left\{x_{0}\right\}} \tilde{f}(x)=1+0=1
$$

by Equation (2), so $E \in O$. Since $Y \subseteq W \subseteq f^{-1}(0) \subseteq \tilde{f}^{-1}(0), Y$ algebraically generates $B(Y)$ and $\tilde{f}$ is a homomorphism, we obtain $\tilde{f}(B(Y)) \subseteq\{0\}$. This shows that $O \cap B(Y)=\varnothing$.

We refer the reader to ([21], Section 2) for properties of free precompact (abelian) groups and [22] for those of free precompact Boolean groups.

\section{Coherent Maps}

Definition 7. Given sets $P \subseteq \beta \mathbb{N} \backslash \mathbb{N}$ and $K$, define $X=P \times K \times(\omega+1)$ and $X^{*}=P \times K \times\{\omega\}$.

Definition 8. Let $X$ be a set as in Definition 7. We shall say that a map $f: X \rightarrow \mathbb{Z}_{2}$ is coherent provided that

$$
\{n \in \omega: f(p, k, n)=f(p, k, \omega)\} \in p \text { for every } p \in P \text { and each } k \in K .
$$

Note that the map $f: X \rightarrow \mathbb{Z}_{2}$ is coherent if and only if $f(p, k, \omega)$ is a $p$-limit of the sequence $\{f(p, k, n): n \in \mathbb{N}\}$ whenever $p \in P$ and $k \in K$.

Definition 9. We introduce the topology on a set $X$ as in Definition 7 by declaring each point of $X \backslash X^{*}$ to be isolated and a basic open neighbourhood of a point $(p, k, \omega) \in X^{*}$ to be of the form $\{(p, k, \omega)\} \cup\{(p, k, n)$ : $n \in F\}$ for a given element $F \in p$.

Remark 6. Let X be a topological space from Definition 9.

(i) Note that $X_{p, k}=\{(p, k, n): n \in \omega+1\}$ for $(p, k) \in P \times K$ is a clopen subset of $X$, so $X=\bigoplus_{(p, k) \in P \times K} X_{p, k}$ is a topological sum of $X_{p, k}$.

(ii) Since each $X_{p, k}$ for $(p, k) \in P \times K$ is a space with a single non-isolated point, it is zero-dimensional. It follows from this and (i) that $X$ is zero-dimensional as well.

The straightforward verification of the following lemma is left to the reader.

Lemma 4. Let $X$ be a set as in Definition 7. Then a map $f: X \rightarrow \mathbb{Z}_{2}$ is coherent in the sense of Definition 8 if and only if it is continuous with respect to the topology on $X$ described in Definition 9 and the discrete topology on $\mathbb{Z}_{2}$.

We finish this section with two technical lemmas which will be needed in future proofs. The reader can safely skip them during the first pass.

Lemma 5. Let $X$ and $X^{*}$ be sets as in Definition 7. Then every map $g: X \backslash X^{*} \rightarrow \mathbb{Z}_{2}$ admits a unique coherent extension $f: X \rightarrow \mathbb{Z}_{2}$ over $X$.

Proof. For fixed $p \in P$ and $k \in K$, we have

$$
\{n \in \omega: g(p, k, n)=0\} \cup\{n \in \omega: g(p, k, n)=1\}=\omega \in p .
$$

Since $p$ is an ultrafilter on $\omega$, there exists a unique $i_{p, k}=0,1$ such that

$$
\left\{n \in \omega: g(p, k, n)=i_{p, k}\right\} \in p .
$$


Define $f(p, k, \omega)=i_{p, k}$ for every $p \in P$ and $k \in K$. Finally, let $f(p, k, n)=g(p, k, n)$ for all $(p, k, n) \in X \backslash X^{*}=P \times K \times \omega$. It follows from this definition and Equation (4) that Equation (3) holds; that is, $f$ is coherent by Definition 8 .

Lemma 6. Let $X$ be a set as in Definition 7. If $P^{\prime} \subseteq P, K^{\prime} \subseteq K$ and $h \in B(X) \backslash B\left(P^{\prime} \times K^{\prime} \times(\omega+1)\right)$, then there exists a coherent map $f: X \rightarrow \mathbb{Z}_{2}$ such that $\tilde{f}\left(B\left(P^{\prime} \times K^{\prime} \times(\omega+1)\right)\right) \subseteq\{0\}$ and $\tilde{f}(h)=1$.

Proof. Fix a finite set $F \subseteq X$ such that $h=\sum_{(p, k, n) \in F}\{(p, k, n)\}$. It follows from $h \in B(X) \backslash B\left(P^{\prime} \times\right.$ $\left.K^{\prime} \times(\omega+1)\right)$ that $F \nsubseteq P^{\prime} \times K^{\prime} \times(\omega+1)$, so we can fix

$$
\left(p_{0}, k_{0}, n_{0}\right) \in F \backslash\left(P^{\prime} \times K^{\prime} \times(\omega+1)\right) .
$$

Since $F$ is finite, there exists $m \in \omega$ such that $\left(p_{0}, k_{0}, n\right) \notin F$ for all $n \in \omega$ with $n \geq m$. Define $f: X \rightarrow \mathbb{Z}_{2}$ by

$$
f(p, k, n)=\left\{\begin{array}{ll}
1 & \text { if } p=p_{0}, k=k_{0} \text { and either } n=n_{0} \text { or } n \geq m \\
0 & \text { otherwise }
\end{array} \quad \text { for }(p, k, n) \in X .\right.
$$

Let $p \in P$ and $k \in K$ be arbitrary. If either $p \neq p_{0}$ or $k \neq k_{0}$, then $f(p, k, n)=0$ for every $n \in \omega+1$ by Equation (6), so $\omega=\{n \in \omega: f(p, k, n)=f(p, k, \omega)=0\} \in p$. Suppose now that $p=p_{0}$ and $k=k_{0}$. Then

$$
\{n \in \omega: n \geq m\} \subseteq\left\{n \in \omega: f\left(p_{0}, k_{0}, n\right)=f\left(p_{0}, k_{0}, \omega\right)=1\right\}=N
$$

by Equation (6). Since $p$ is a free ultrafilter on $\omega$, we have $\{n \in \omega: n \geq m\} \in p$. This implies that $N \in p$, and therefore, $f$ is coherent by Definition 8 .

If $(p, k, n) \in P^{\prime} \times K^{\prime} \times(\omega+1)$, then either $p \neq p_{0}$ or $k \neq k_{0}$ by Equation (5), so $f(p, k, n)=0$ by Equation (6). Therefore, $f\left(P^{\prime} \times K^{\prime} \times(\omega+1)\right) \subseteq\{0\}$. Since $\tilde{f}$ is a homomorphism extending $f$, it easily follows that $\tilde{f}\left(B\left(P^{\prime} \times K^{\prime} \times(\omega+1)\right)\right) \subseteq\{0\}$.

From the choice of $m$ and Equation (6), we conclude that $f(p, k, n)=0$ for all $(p, k, n) \in F \backslash$ $\left\{\left(p_{0}, k_{0}, n_{0}\right)\right\}$. Furthermore, $f\left(p_{0}, k_{0}, n_{0}\right)=1$ by Equation (6).

Since $\tilde{f}$ is a homomorphism extending $f$, we obtain

$$
\tilde{f}(h)=\tilde{f}\left(\sum_{(p, k, n) \in F}\{(p, k, n)\}\right)=\sum_{(p, k, n) \in F} \tilde{f}\{(p, k, n)\}=\sum_{(p, k, n) \in F} f(p, k, n)=f\left(p_{0}, k_{0}, n_{0}\right)=1 .
$$

This finishes the proof of our lemma.

\section{Coherent Splitting Maps and Their Continuity}

Definition 10. Let $X$ be a set. We shall say that a map $f: X \rightarrow \mathbb{Z}_{2}$ splits a subset $A$ of $B(X)$ provided that the set $\{a \in A: \tilde{f}(a)=i\}$ is infinite for each $i \in \mathbb{Z}_{2}$, where $\tilde{f}: B(X) \rightarrow \mathbb{Z}_{2}$ is the homomorphism defined in Equation (2).

Clearly, a subset split by some map must be infinite. The converse also holds:

Lemma 7. For an arbitrary set $X$, every infinite subset of $B(X)$ can be split by some map $f: X \rightarrow \mathbb{Z}_{2}$.

This lemma is part of folklore and can be proved by a straightforward induction. It can also be derived from ([23], Lemma 4.1).

The secondary goal of this paper is to prove the following theorem strengthening Lemma 7 by additionally requiring the splitting map to be coherent. 
Theorem 2. If $X$ is a set as in Definition 7 , then every infinite subset of $B(X)$ can be split by some coherent map $f: X \rightarrow \mathbb{Z}_{2}$.

This theorem constitutes the main technical tool in the proof of Theorem 1 in Section 10. The proof of Theorem 2 is postponed until Section 9.

The next corollary provides a topological reformulation of Theorem 2.

Corollary 6. Let $X$ be a set as in Definition 7 equipped with the topology described in Definition 9. Then for every infinite subset $A$ of the free precompact Boolean group $F P B(X)$ of $X$, there exists a continuous homomorphism $\pi: \operatorname{FPB}(X) \rightarrow \mathbb{Z}_{2}$ such that the set $\{a \in A: \pi(a)=i\}$ is infinite for each $i \in \mathbb{Z}_{2}$.

Proof. In this proof, we use notations from Lemma 2. The space $X$ is zero-dimensional by Remark 6 (ii). By Lemma 2, we can identify $F P B(X)$ with $\left(B(X), \mathscr{T}_{X}\right)$. After this identification, we can think of $A$ as being an infinite subset of $B(X)$. By Theorem 2, $A$ is split by some coherent map $f: X \rightarrow \mathbb{Z}_{2}$. By Lemma $4, f$ is continuous, and so $f \in \mathscr{F}_{X}$, which implies that $\pi=\tilde{f} \in \tilde{\mathscr{F}}_{X}$. Since $\mathscr{T}_{X}$ is the initial topology with respect to the family $\tilde{\mathscr{F}}_{X}$, the map $\pi$ is $\mathscr{T}_{X}$-continuous. Recalling our identification of $F P B(X)$ with $\left(B(X), \mathscr{T}_{X}\right)$, we conclude that the homomorphism $\pi: F P B(X) \rightarrow \mathbb{Z}_{2}$ is continuous. Since $A$ is split by $f$, it follows from this and Definition 10 that $\pi$ satisfies the conclusion of our corollary.

Definition 11. For simplicity, we shall say that a topological space is elementary if it is homeomorphic to a subspace of $\beta \mathbb{N}$ of the form $\mathbb{N} \cup\{p\}$, where $p \in \beta \mathbb{N} \backslash \mathbb{N}$.

Corollary 7. Let $K$ be a non-empty set. For every $k \in K$, let $Y_{k}$ be either an at most countable discrete space or an elementary space. Let $Y=\bigoplus_{k \in K} Y_{k}$ be the topological sum of the family $\left\{Y_{k}: k \in K\right\}$. Then for every infinite subset $A$ of $F P B(Y)$, there exists a continuous homomorphism $h: F P B(Y) \rightarrow \mathbb{Z}_{2}$ such that the set $\{a \in A: h(a)=i\}$ is infinite for each $i \in \mathbb{Z}_{2}$.

Proof. Let $P=\beta \mathbb{N} \backslash \mathbb{N}$ and let $X=P \times K \times(\omega+1)$ be the set as in Definition 7 . We equip $X$ with the topology described in Definition 9. In this proof, we use notations from Remark 6 (i).

Fix a free ultrafilter $q$ on $\mathbb{N}$. Let $k \in K$. If $Y_{k}$ is an at most countable discrete space, then we can fix an injection $\varphi_{k}: Y_{k} \rightarrow X_{q, k}$ which will obviously be continuous. If $Y_{k}$ is an elementary space, then Definition 11 allows us to identify the space $Y_{k}$ with the subspace $\mathbb{N} \cup\left\{p_{k}\right\}$ of $\beta \mathbb{N}$, for a suitable $p_{k} \in \beta \mathbb{N} \backslash \mathbb{N}$. Now we can fix an injection $\varphi_{k}: Y_{k} \rightarrow X_{p_{k}, k}$ which sends each point $n \in \mathbb{N}$ to the point $\left(p_{k}, k, n\right) \in X_{p_{k}, k}$ and the point $p_{k} \in Y_{k}$ to $\left(p_{k}, k, \omega\right) \in X_{p_{k}, k}$. Clearly, $\varphi_{k}$ is a homeomorphism between $Y_{k}$ and $X_{p_{k}, k}$.

Let $\varphi: Y=\bigoplus_{k \in K} Y_{k} \rightarrow X$ be the map such that $\varphi\left\lceil Y_{k}=\varphi_{k}\right.$ for every $k \in K$. Since each $\varphi_{k}$ is an injection, so is $\varphi$. Since each $\varphi_{k}$ is continuous, it follows from our definition of $\varphi$ and Remark 6 (i) that $\varphi$ is continuous as well.

Clearly, $Y$ is zero-dimensional, and $X$ is zero-dimensional by Remark 6 (ii). Since $\varphi: Y \rightarrow X$ is a continuous injection, $\hat{\varphi}: F P B(Y) \rightarrow F P B(X)$ is a continuous monomorphism by Lemma 3 (i).

Let $A$ be an infinite subset of $F P B(Y)$. Then $B=\hat{\varphi}(A)$ is an infinite subset of $F P B(X)$. By Corollary 6, we can find a continuous homomorphism $\pi: F P B(X) \rightarrow \mathbb{Z}_{2}$ such that the set $\{b \in B: \pi(b)=i\}$ is infinite for each $i \in \mathbb{Z}_{2}$. Now the composition $h=\pi \circ \hat{\varphi}: F P B(Y) \rightarrow \mathbb{Z}_{2}$ is the desired homomorphism, as $\hat{\varphi} \uparrow_{A}: A \rightarrow B$ is a one-to-one map.

\section{Applications to Free Precompact Boolean Groups of Topological Sums of Maximal Spaces}

Definition 12. Recall that a space is maximal if it is non-discrete, yet any strictly stronger topology on it is discrete. 
One easily sees that every maximal space $X$ has exactly one non-isolated point $p$ such that the trace of the filter of neighbourhoods of $p$ on the set $D=X \backslash\{p\}$ of isolated points of $X$ is an ultrafilter on $D$. In particular, $X$ is zero-dimensional.

Clearly, elementary spaces from Definition 11 are precisely the countably infinite maximal spaces.

Lemma 8. Let $X$ be either a discrete or a maximal topological space, and let $Y$ be an at most countable closed subspace of $X$. Then

(i) $Y$ is either elementary or discrete, and

(ii) $Y$ is $\mathbb{Z}_{2}$-embedded in $X$.

Proof. The conclusion of our lemma is trivial when $X$ is a discrete space. Therefore, from now on we shall assume that $X$ is a maximal space. Let $p$ be the non-isolated point of $X$. We consider two cases.

Case 1. $p \in Y$. If $p$ is a non-isolated point in $Y$, then every neighbourhood of $p$ intersects the set $Y \backslash\{p\}$. By the maximality of $X$, we conclude that $Y$ is a neighbourhood of $p$ in $X$. This means that $Y$ is clopen in $X$, and therefore $\mathbb{Z}_{2}$-embedded in $X$ by Remark 5 . Applying maximality of $X$ once again, we conclude that $Y$ is an elementary space.

Suppose now that $p$ is an isolated point of $Y$. Then $Y$ is discrete and there exists an open subset $U$ of $X$ such that $U \cap Y=\{p\}$. If $g: Y \rightarrow \mathbb{Z}_{2}$ is a continuous map, then the map $f: X \rightarrow \mathbb{Z}_{2}$ defined by

$$
f(x)= \begin{cases}g(x) & \text { if } x \in Y \backslash\{p\} \\ g(p) & \text { if } x \in U \\ 0 & \text { otherwise }\end{cases}
$$

is continuous and extends $g$. This shows that $Y$ is $\mathbb{Z}_{2}$-embedded in $X$.

Case 2. $p \in X \backslash Y$. Since $p$ is the only non-isolated point of $X$, all points of $Y$ are isolated in $X$, so $Y$ is discrete and open in $X$. Since $Y$ is also closed in $X$, it is clopen in $X$, and so $\mathbb{Z}_{2}$-embedded in $X$ by Remark 5.

Lemma 9. Let $X=\bigoplus_{j \in J} X_{j}$ be the topological sum of a family $\left\{X_{j}: j \in J\right\}$, where each space $X_{j}$ is either discrete or maximal. Let $A$ be an at most countable subset of $F P B(X)$. Then there exist an at most countable set $K \subseteq J$ and an at most countable closed subspace $Y_{k}$ of $X_{k}$ for each $k \in K$ such that the topological sum $Y=\bigoplus_{k \in K} Y_{k}$ satisfies the following conditions:

(i) each $Y_{k}$ is either elementary or discrete;

(ii) $F P B(Y)$ is an at most countable closed subgroup of $F P B(X)$;

(iii) every continuous homomorphism $h: F P B(Y) \rightarrow \mathbb{Z}_{2}$ can be extended to a continuous homomorphism $\varphi: F P B(X) \rightarrow \mathbb{Z}_{2}$

(iv) $A \subseteq F P B(Y)$.

Proof. Since $X$ is zero-dimensional, Lemma 2 allows us to identify $F P B(X)$ with $\left(B(X), \mathscr{T}_{X}\right)$, so we can view $A$ as a subset of $B(X)$. Since $A$ is countable, there exists an at most countable set $S \subseteq X$ such that $A \subseteq B(S)$. Since $X=\bigoplus_{j \in J} X_{j}$, we can find an at most countable set $K \subseteq J$, and for every $k \in K$ we can fix an at most countable subset $Y_{k}$ of $X_{k}$ such that $S \subseteq Y$, where $Y=\bigoplus_{k \in K} Y_{k}$. Without loss of generality, we may assume that each $Y_{k}$ contains the unique non-isolated point of $X_{k}$ whenever $X_{k}$ is a maximal space. This assumption means that $Y_{k}$ is closed in $X_{k}$ for each $k \in K$.

(i) By Lemma 8 , each space $Y_{k}$ is either elementary or discrete, and $Y_{k}$ is $\mathbb{Z}_{2}$-embedded in $X_{k}$.

(ii) Since $Y_{k}$ is a closed $\mathbb{Z}_{2}$-embedded subspace of $X_{k}$ for every $k \in K$, we conclude that $Y$ is a closed $\mathbb{Z}_{2}$-embedded subspace of $X$. Therefore, $F P B(Y)$ is a closed subgroup of $F P B(X)$ by Lemma 3 (ii). Since $Y$ is zero-dimensional, Lemma 2 allows us to identify $F P B(Y)$ with $\left(B(Y), \mathscr{T}_{Y}\right)$. Since $Y$ is at most countable, so is $B(Y)$ and thus $F P B(Y)$. 
(iii) Let $h: F P B(Y) \rightarrow \mathbb{Z}_{2}$ be an arbitrary continuous homomorphism. Since the topology of $F P B(Y)$ induces the original topology of $Y$ by Lemma 2, the restriction $g=h \uparrow_{Y}: Y \rightarrow \mathbb{Z}_{2}$ of $h$ to $Y$ is continuous. Since $Y$ is $\mathbb{Z}_{2}$-embedded in $X$, we can find a continuous map $f: X \rightarrow \mathbb{Z}_{2}$ extending $g$. Since $F P B(X)$ coincides with $\left(B(X), \mathscr{T}_{X}\right)$ and $F P B(Y)$ coincides with $\left(B(Y), \mathscr{T}_{Y}\right)$, it follows that $\varphi=\tilde{f}$ is a continuous homomorphism from $F P B(X)$ to $\mathbb{Z}_{2}$ whose restriction to $F P B(Y)$ coincides with $h=\tilde{g}$.

(iv) Since $A \subseteq B(S)$ and $S \subseteq Y$, we have $A \subseteq B(S) \subseteq B(Y)$. Therefore, we can view $A$ as a subset of $F P B(Y)$.

Theorem 3. Let $X=\bigoplus_{j \in J} X_{j}$ be the topological sum of a family $\left\{X_{j}: j \in J\right\}$, where each space $X_{j}$ is either discrete or maximal. Then for every infinite subset $A$ of $F P B(X)$, there exists a continuous homomorphism $\varphi: F P B(X) \rightarrow \mathbb{Z}_{2}$ such that the set $\{a \in A: \varphi(a)=i\}$ is infinite for each $i \in \mathbb{Z}_{2}$.

Proof. Without loss of generality, we may assume that $A$ is countably infinite. Applying Lemma 9 to this $A$, we can obtain a subspace $Y$ of $X$ as in the conclusion of Lemma 9. By Item (i) of this lemma, we can apply Corollary 7 to find a continuous homomorphism $h: F P B(Y) \rightarrow \mathbb{Z}_{2}$ such that the set $\{a \in A: h(a)=i\}$ is infinite for each $i \in \mathbb{Z}_{2}$. Applying Item (iii) of Lemma 9, we can find a continuous homomorphism $\varphi: F P B(X) \rightarrow \mathbb{Z}_{2}$ extending $h$. Since $A \subseteq F P B(Y)$ by Item (iv) of Lemma 9, we have $\{a \in A: \varphi(a)=i\}=\{a \in A: h(a)=i\}$ for each $i \in \mathbb{Z}_{2}$.

Lemma 10. Let $X$ be a topological space such that the closure of each at most countable subset of $X$ is at most countable. Then every separable pseudocompact subspace $\mathrm{K}$ of $\mathrm{X}$ is compact and metrizable. Moreover, if $\mathrm{K}$ is infinite, then $K$ contains a non-trivial convergent sequence.

Proof. Let $K$ be a separable pseudocompact subset of $X$. Let $S$ be an at most countable dense subset of $K$. Then its closure $C$ in $X$ is at most countable by the assumption of our lemma. Since $S$ is dense in $K$, we have $K \subseteq C$. Thus, $K$ is an at most countable pseudocompact space, so it must be compact. An at most countable compact space is metrizable [24], so $K$ is a metrizable compact space. The last sentence of our lemma follows from the fact that every infinite compact metrizable space has a non-trivial convergent sequence.

Theorem 4. Let $X=\bigoplus_{j \in J} X_{j}$ be the topological sum of a family $\left\{X_{j}: j \in J\right\}$, where each space $X_{j}$ is either discrete or maximal. Let $G=F P B(X)$ be the free precompact Boolean group of $X$. Then all separable pseudocompact subsets of $G$ are finite.

Proof. First, we check that each at most countable subset $A$ of $G=F P B(X)$ has at most countable closure in G. If $A$ is finite, then it is closed in G. Suppose now that $A$ is infinite. Applying Lemma 9 to this $A$, we can obtain a subspace $Y$ of $X$ as in the conclusion of Lemma 9. By Item (ii) of this lemma, $H=F P B(Y)$ is an at most countable closed subgroup of $F P B(X)=G$. Note that $A \subseteq H$ by Item (iv) of Lemma 9. Therefore, the closure of $A$ in $G$ is contained in the (at most countable) set $H$.

Let $A$ be a countably infinite subset of G. Applying Theorem 3, we can find a continuous homomorphism $\varphi: G \rightarrow \mathbb{Z}_{2}$ such that the set $\{a \in A: \varphi(a)=i\}$ is infinite for each $i \in \mathbb{Z}_{2}$. Since $\varphi$ is continuous, $A_{i}=\{a \in A: \varphi(a)=i\}$ is a closed subset of $A$ for $i \in \mathbb{Z}_{2}=\{0,1\}$. Since $A=A_{0} \cup A_{1}$ is a partition of $A$ into two disjoint infinite closed sets, $A$ cannot be a convergent sequence. We have proved that $G$ does not contain non-trivial convergent sequences. By Lemma 10, all separable pseudocompact subsets of $G$ are finite.

The group $G=F P B(X)$ in Theorem 4 is precompact, so its completion $H$ is a compact group. Being compact, the group $H$ contains many non-trivial convergent sequences. Since these non-trivial convergent sequences in $H$ might appear already in its subgroup $G$, this demonstrates that Theorem 4 is not completely trivial. 


\section{Discussion}

The topic of this paper is related to a long-standing open problem of van Douwen about the existence in ZFC alone of a countably compact group without non-trivial convergent sequences. (The existence of such a group in some additional set-theoretic axioms, such as Continuum Hypothesis (CH) or Martin's Axiom (MA), is well-known.) Indeed, it was noted in ([5], Example 5.7) that a solution to this problem would bring a positive solution to Question 1 (ii) and thus to the weaker Question 1 (i).

The question of the existence of pseudocompact groups without infinite compact subsets (and its weaker version which only prohibits non-trivial convergent sequences) has been studied extensively [12-14,25,26]. For example, Galindo and Macario proved that, under a mild additional set-theoretic assumption beyond ZFC, every pseudocompact abelian group admits a pseudocompact group topology without infinite compact subsets [12]. Corollary 1 contributes to this topic by constructing an abelian topological group without infinite compact subsets (in fact, even without infinite separable pseudocompact subsets) which has a much stronger property than mere pseudocompactness.

Topological groups without infinite compact subsets play a prominent role in Pontryagin duality theory [27] due to the fact that pseudocompact abelian groups without infinite compact subsets are (Pontryagin) reflexive ([11], Theorem 2.8) (this also follows from ([12], Lemma 2.3 and Theorem 6.1)). All topological groups we construct in this paper are reflexive by Remark 3.

The strongest precompact group topology on an abelian group is called its Bohr topology. It is a classical result of Glicksberg that the Bohr topology on any abelian group does not have infinite compact subsets [28]; see also ([29], Section 6) for an alternative proof. Since the free precompact Boolean group $F P B(X)$ of a topological space $X$ is precompact, its topology $\mathscr{T}_{X}$ is weaker than the corresponding Bohr topology, so $\mathscr{T}_{X}$ can have more compact subsets than the Bohr topology (in which all compact subsets are finite). Note that, when $X$ is discrete, then $\mathscr{T}_{X}$ coincides with the Bohr topology on $F P B(X)$, so it does not have infinite compact subsets by Glicksberg's result. Our Theorem 4 can be viewed as an extension of Glicksberg's theorem over free precompact Boolean groups $F P B(X)$ of spaces $X$ very close to being discrete (indeed, maximal spaces are one step from being discrete by Definition 12).

The idea of splitting of a given infinite subset $A$ of a discrete abelian group $G$ via a homomorphism $\varphi$ from $G$ to some target topological group $H$ (usually $\mathbb{Z}_{2}$ or the torus group $\mathbb{T}$ ) is a classical technique for producing a group topology on $G$ without non-trivial convergent sequences. Such a splitting is always possible, modulo natural algebraic restrictions on $H$ and $A$; see $[23,29,30]$. However, if $G$ is equipped with a non-discrete group topology $\mathscr{T}$, finding a $\mathscr{T}$-continuous homomorphism $\varphi$ which splits $A$ is a much more difficult task, and the authors are not aware of any known results in this direction. Therefore, our Theorem 3 can be viewed as a first, albeit somewhat modest, contribution to what is undoubtedly quite an interesting topic.

\section{Proof of Theorem 2}

In this section, we fix a non-empty set $P \subseteq \beta \mathbb{N} \backslash \mathbb{N}$, a non-empty set $K$ and consider sets

$$
X=P \times K \times(\omega+1) \text { and } X^{*}=P \times K \times\{\omega\}
$$

from Definition 7. We also fix an infinite subset $A$ of $B(X)$.

Lemma 11. If $X^{*} \cap(\bigcup A)$ is finite, then some coherent map $f: X \rightarrow \mathbb{Z}_{2}$ splits $A$.

Proof. Since $J=X^{*} \cap(\bigcup A)$ is finite and $A$ is infinite, there exists $I \in[J]^{<\omega}$ such that the set

$$
A^{\prime}=\left\{a \in A: a \cap X^{*}=I\right\}
$$

is infinite. Then

$$
B=\left\{a \backslash X^{*}: a \in A^{\prime}\right\}=\left\{a \backslash I: a \in A^{\prime}\right\}
$$


is an infinite subset of $B\left(X \backslash X^{*}\right)$. By Lemma 7, there exists a map $g: X \backslash X^{*} \rightarrow \mathbb{Z}_{2}$ which splits $B$. Let $f: X \rightarrow \mathbb{Z}_{2}$ be the unique coherent map extending $g$ given by Lemma 5. Clearly, $\tilde{f} \uparrow_{B\left(X \backslash X^{*}\right)}=\tilde{g}$. Since $B \subseteq B\left(X \backslash X^{*}\right)$ and $g$ splits $B$, the map $f$ splits $B$ as well. It follows from this, Equation (8), and Definition 10 that

$$
\left\{a \in A^{\prime}: \tilde{f}(a \backslash I)=i\right\} \text { is infinite for every } i \in \mathbb{Z}_{2} .
$$

Define $j=\tilde{f}(I)$. Clearly, $j \in \mathbb{Z}_{2}$. It follows from Equations (7) and (8) that $a=(a \backslash I) \cup I$ for every $a \in A^{\prime}$, so $a=(a \backslash I)+I$ holds in $B(X)$; therefore,

$$
\tilde{f}(a)=\tilde{f}(a \backslash I)+\tilde{f}(I)=\tilde{f}(a \backslash I)+j \text { for } a \in A^{\prime},
$$

as $\tilde{f}$ is a homomorphism. Combining Equations (9) and (10), we conclude that $\left\{a \in A^{\prime}: \tilde{f}(a)=i\right\}$ is infinite for every $i \in \mathbb{Z}_{2}$. Since $A^{\prime} \subseteq A$, the same conclusion holds when $A^{\prime}$ is replaced by $A$. According to Definition 10, this means that $f$ splits $A$.

Definition 13. We denote by $\mathbb{Q}$ the set of all triples $q=\left\langle P^{q}, K^{q}, f^{q}\right\rangle$, where $P^{q} \in[P]^{<\omega}, K^{q} \in[K]^{<\omega}$ and $f^{q}: P^{q} \times K^{q} \times(\omega+1) \rightarrow \mathbb{Z}_{2}$ is a coherent map. For $q=\left\langle P^{q}, K^{q}, f^{q}\right\rangle, r=\left\langle P^{r}, K^{r}, f^{r}\right\rangle \in \mathbb{Q}$, we let $q \leq r$ provided that $P^{r} \subseteq P^{q}, K^{r} \subseteq K^{q}$, and $f^{q}$ extends $f^{r}$.

One easily sees that $(\mathbb{Q}, \leq)$ is a poset. Clearly, $\langle\varnothing, \varnothing, \varnothing\rangle \in \mathbb{Q}$, so $\mathbb{Q} \neq \varnothing$.

Recall that a set $D \subseteq \mathbb{Q}$ is said to be dense in $(\mathbb{Q}, \leq)$ provided that for every $r \in \mathbb{Q}$ there exists $q \in D$ such that $q \leq r$.

Lemma 12. (i) For every $p \in P$, the set $C_{p}=\left\{q \in \mathbb{Q}: p \in P^{q}\right\}$ is dense in $(\mathbb{Q}, \leq)$.

(ii) For every $k \in K$, the set $E_{k}=\left\{q \in \mathbb{Q}: k \in K^{q}\right\}$ is dense in $(\mathbb{Q}, \leq)$.

Proof. (i) Suppose that $r \in \mathbb{Q} \backslash C_{p}$. Then $p \in P \backslash P^{r}$. Note that the extension $f^{q}: P^{q} \times K^{q} \times(\omega+1) \rightarrow$ $\mathbb{Z}_{2}$ of $f^{r}$, obtained by letting $f^{q}(p, k, n)=0$ for all $k \in K^{q}=K^{r}$ and $n \in \omega+1$, is coherent. Then $q=\left\langle P^{q}, K^{q}, f^{q}\right\rangle \in \mathbb{Q}$. Clearly, $q \in C_{p}$ and $q \leq r$.

(ii) Suppose that $r \in \mathbb{Q} \backslash E_{k}$. Then $k \in K \backslash K^{r}$. Define $P^{q}=P^{r}$ and $K^{q}=K^{r} \cup\{k\}$. Note that the extension $f^{q}: P^{q} \times K^{q} \times(\omega+1) \rightarrow \mathbb{Z}_{2}$ of $f^{r}$, obtained by letting $f^{q}(p, k, n)=0$ for all $p \in P^{q}=P^{r}$ and $n \in \omega+1$, is coherent. Then $q=\left\langle P^{q}, K^{q}, f^{q}\right\rangle \in \mathbb{Q}$. Clearly, $q \in E_{k}$ and $q \leq r$.

Lemma 13. If $X^{*} \cap(\cup A)$ is infinite, then for every $B \in[A]^{<\omega}$ and each $i \in \mathbb{Z}_{2}$, the set

$$
D_{B, i}=\left\{q \in \mathbb{Q}: \exists a \in A \backslash B\left(a \subseteq P^{q} \times K^{q} \times(\omega+1) \text { and } \tilde{f^{q}}(a)=i\right)\right\}
$$

is dense in $(\mathbb{Q}, \leq)$.

Proof. Let $r \in \mathbb{Q}, B \in[A]^{<\omega}$, and $i \in \mathbb{Z}_{2}$ be arbitrary. We need to find $q \in \mathbb{Q}$ and $a \in A \backslash B$ such that $q \leq r, a \subseteq P^{q} \times K^{q} \times(\omega+1)$, and $\tilde{f}^{q}(a)=i$.

Since $B$ is finite, the intersection $X^{*} \cap(\cup B)$ is also finite. Furthermore, since both $P^{r}$ and $K^{r}$ are finite sets, so is the set $P^{r} \times K^{r} \times\{\omega\}$. Therefore,

$$
F=\left(X^{*} \cap(\bigcup B)\right) \cup\left(P^{r} \times K^{r} \times\{\omega\}\right)
$$

is a finite subset of $X^{*}$. By our hypothesis, $X^{*} \cap(\bigcup A)$ is infinite, so there exists $a \in A$ such that $\left(a \cap X^{*}\right) \backslash F \neq \varnothing$. Fix $p_{0} \in P, k_{0} \in K$, and $a \in A$ such that $\left(p_{0}, k_{0}, \omega\right) \in a \backslash F$. It follows from this and Equation (12) that $a \in A \backslash B$.

Since $a$ is a finite subset of $X=P \times K \times(\omega+1)$, there exist finite sets $P^{q} \subseteq P$ and $K^{q} \subseteq K$ such that $a \subseteq P^{q} \times K^{q} \times(\omega+1)$. By Lemma 12, without loss of generality, we may also assume that $P^{r} \subseteq P^{q}$ and $K^{r} \subseteq K^{q}$. 
Let $a^{\prime}=a \cap\left(P^{r} \times K^{r} \times(\omega+1)\right)$. Then $j=\tilde{f}^{r}\left(a^{\prime}\right) \in \mathbb{Z}_{2}$ is well-defined. There exists a unique $l \in \mathbb{Z}_{2}$ such that $j+l=i$. Note that $\left(p_{0}, k_{0}\right) \in\left(P^{q} \times K^{q}\right) \backslash\left(P^{r} \times K^{r}\right)$, so we can define a map $f^{q}: P^{q} \times K^{q} \times(\omega+1) \rightarrow \mathbb{Z}_{2}$ by

$$
f^{q}(p, k, n)= \begin{cases}f^{r}(p, k, n) & \text { if }(p, k, n) \in P^{r} \times K^{r} \times(\omega+1) \\ l & \text { if }(p, k)=\left(p_{0}, k_{0}\right) \text { and either } n=\omega \text { or }(p, k, n) \notin a \\ 0 & \text { otherwise }\end{cases}
$$

for all $(p, k, n) \in P^{q} \times K^{q} \times(\omega+1)$.

Claim 1. $q=\left\langle P^{q}, K^{q}, f^{q}\right\rangle \in \mathbb{Q}$ and $q \leq r$.

Proof. Since $P^{q} \in[P]^{<\omega}$ and $K^{q} \in[K]^{<\omega}$ by our construction, we only need to check that the map $f^{q}: P^{q} \times K^{q} \times(\omega+1) \rightarrow \mathbb{Z}_{2}$ is coherent. Let $p \in P^{q}$ and $k \in K^{q}$ be arbitrary. If $(p, k) \in P^{r} \times K^{r}$, then

$$
\left\{n \in \omega: f^{q}(p, k, n)=f^{q}(p, k, \omega)\right\}=\left\{n \in \omega: f^{r}(p, k, n)=f^{r}(p, k, \omega)\right\} \in p
$$

by Equation (13) and coherency of $f^{r}$. Suppose now that $(p, k) \in\left(P^{q} \times K^{q}\right) \backslash\left(P^{r} \times K^{r}\right)$. If $(p, k) \neq$ $\left(p_{0}, k_{0}\right)$, then $f^{q}(p, k, n)=0$ for all $n \in \omega+1$ by Equation (13), so $\left\{n \in \omega: f^{q}(p, k, n)=f^{q}(p, k, \omega)=\right.$ $0\}=\omega \in p$. Finally, if $(p, k)=\left(p_{0}, k_{0}\right)$, then the second line of Equation (13) implies that $f^{q}(p, k, \omega)=l$ and $f^{q}(p, k, n)=l$ for all but finitely many $n \in \omega$, as the set $a$ is finite. Therefore, $\left\{n \in \omega: f^{q}(p, k, n)=\right.$ $\left.f^{q}(p, k, \omega)\right\}$ is a cofinite subset of $\omega$, so it belongs to $p$, as $p$ is a free ultrafilter on $\omega$. This finishes the check of the inclusion $q \in \mathbb{Q}$.

Finally, note that $f^{q}$ extends $f^{r}$ by the first line of Equation (13). It follows from this, $P^{r} \subseteq P^{q}$, $K^{r} \subseteq K^{q}$, and Definition 13 that $q \leq r$.

Claim 2. $\tilde{f}^{q}\left(a \backslash a^{\prime}\right)=l$.

Proof. Since $a^{\prime}=a \cap\left(P^{r} \times K^{r} \times(\omega+1)\right)$, we have $a \backslash a^{\prime} \subseteq\left(\left(P^{q} \times K^{q}\right) \backslash\left(P^{r} \times K^{r}\right)\right) \times(\omega+1)$, so Equation (13) implies that $f^{q}\left(p_{0}, k_{0}, \omega\right)=l$ and $f^{q}(p, k, n)=0$ for all $(p, k, n) \in a \backslash\left(a^{\prime} \cup\left\{\left(p_{0}, k_{0}, \omega\right)\right\}\right)$. Since $\tilde{f}^{q}$ is a homomorphism and $\left(p_{0}, k_{0}, \omega\right) \in a \backslash a^{\prime}$ by our choice, this implies

$$
\tilde{f}^{q}\left(a \backslash a^{\prime}\right)=\sum_{(p, k, n) \in a \backslash a^{\prime}} \tilde{f^{q}}(\{p, k, n\})=\sum_{(p, k, n) \in a \backslash a^{\prime}} f^{q}(p, k, n)=f^{q}\left(p_{0}, k_{0}, \omega\right)=l .
$$

This establishes our claim.

Claim 3. $q \in D_{B, i}$.

Proof. The only condition in Equation (11) that remains to be checked is the equality $\tilde{f} q(a)=i$. Since $a^{\prime} \subseteq P^{r} \times K^{r} \times(\omega+1) \subseteq P^{q} \times K^{q} \times(\omega+1)$, we have $\tilde{f}^{q}\left(a^{\prime}\right)=\tilde{f}^{r}\left(a^{\prime}\right)=j$. Note that $a=\left(a \backslash a^{\prime}\right) \cup a^{\prime}$, so $a=\left(a \backslash a^{\prime}\right)+a^{\prime}$. Since $\tilde{f}^{q}$ is a homomorphism, $\tilde{f}^{q}(a)=\tilde{f} q\left(a \backslash a^{\prime}\right)+\tilde{f}^{q}\left(a^{\prime}\right)=l+j=i$ by Claim 2.

Since $r \in \mathbb{Q}$ was chosen arbitrarily, the conclusion of our lemma follows from Claims 1 and 3.

We shall need the following folklore lemma.

Lemma 14. If $\mathscr{D}$ is an at most countable family of dense subsets of a non-empty poset $(\mathbb{Q}, \leq)$, then there exists an at most countable subset $\mathbb{F}$ of $\mathbb{Q}$ such that $(\mathbb{F}, \leq)$ is a linearly ordered set and $\mathbb{F} \cap D \neq \varnothing$ for every $D \in \mathscr{D}$.

Proof. Since the family $\mathscr{D}$ is at most countable, we can fix an enumeration $\mathscr{D}=\left\{D_{n}: n \in \mathbb{N} \backslash\{0\}\right\}$ of elements of $\mathscr{D}$. Since $\mathbb{Q} \neq \varnothing$, there exists $q_{0} \in \mathbb{Q}$. By induction on $n \in \mathbb{N} \backslash\{0\}$, we can choose $q_{n} \in D_{n}$ 
such that $q_{n} \leq q_{n-1}$; this is possible because $D_{n}$ is dense in $(\mathbb{Q}, \leq)$. Now $\mathbb{F}=\left\{q_{n}: n \in \mathbb{N} \backslash\{0\}\right\}$ is the desired subset of $\mathbb{Q}$.

Lemma 15. If $P$ and $K$ are at most countable sets and $X^{*} \cap(\cup A)$ is infinite, then some coherent map $f: X \rightarrow \mathbb{Z}_{2}$ splits $A$.

Proof. By Lemmas 12 and 13, the family

$$
\mathscr{D}=\left\{C_{p}: p \in P\right\} \cup\left\{E_{k}: k \in K\right\} \cup\left\{D_{B, i}: B \in[A]^{<\omega}, i \in \mathbb{Z}_{2}\right\}
$$

consists of dense subsets of $(\mathbb{Q}, \leq)$. Since $P, K$, and $A$ are at most countable, so is $\mathscr{D}$. By Lemma 14 , there exists a set $\mathbb{F}=\left\{q_{n}: n \in \mathbb{N}\right\} \subseteq \mathbb{Q}$ such that $q_{0} \geq q_{1} \geq \cdots \geq q_{n} \geq q_{n+1} \geq \ldots$ and $\mathbb{F} \cap D \neq \varnothing$ for every $D \in \mathscr{D}$.

We claim that $f=\bigcup\left\{f^{q_{n}}: n \in \mathbb{N}\right\}$ is a coherent map from $X$ to $\mathbb{Z}_{2}$ splitting $A$. Since $\mathbb{F}$ intersects each $C_{p}$ and every $E_{k}$, the domain of $f$ coincides with $X=P \times K \times(\omega+1)$. Since each $f^{q_{n}}$ is coherent and $f$ extends all $f^{q_{n}}$, it easily follows that $f$ is coherent as well.

Suppose that $f$ does not split $A$. Then the set $B=\{a \in A: \tilde{f}(a)=i\}$ must be finite for some $i \in \mathbb{Z}_{2}$, so $B \in[A]^{<\omega}$ and thus $D_{B, i} \in \mathscr{D}$. Therefore, $q_{n} \in D_{B, i}$ for some $n \in \mathbb{N}$. Applying Equation (11), we can find $a \in A \backslash B$ such that $a \subseteq P^{q_{n}} \times K^{q_{n}} \times(\omega+1)$ and $f^{q_{n}}(a)=i$. Since $f^{q_{n}} \subseteq f$, this implies $\tilde{f}(a)=f \tilde{q}_{n}(a)=i$. Therefore, $a \in B$ by the definition of the set $B$, in contradiction with $a \in A \backslash B$.

Proof of Theorem 2. Let $A$ be an infinite subset of $B(X)$. Choose a countably infinite subset $A^{\prime}$ of $A$. Since $A^{\prime} \subseteq B(X)=[X]^{<\omega}$, there exist at most countable sets $P^{\prime} \subseteq P$ and $K^{\prime} \subseteq K$ such that $A \subseteq B\left(X^{\prime}\right)$, where $X^{\prime}=P^{\prime} \times K^{\prime} \times(\omega+1)$. Combining Lemmas 11 and 15 , we can find a coherent map $f^{\prime}: X^{\prime} \rightarrow \mathbb{Z}_{2}$ splitting $A^{\prime}$. Let $f: X \rightarrow \mathbb{Z}_{2}$ be the extension of $f^{\prime}$ over $X$ obtained by letting $f$ take 0 everywhere on $X \backslash X^{\prime}$. Clearly, $f$ is a coherent map which splits $A^{\prime}$. Since $A^{\prime} \subseteq A, f$ splits $A$ as well.

\section{Proof of Theorem 1}

The following lemma is part of set-theoretic folklore. We include its proof only for convenience of the reader.

Lemma 16. Let $S$ and $T$ be sets such that $1 \leq|S| \leq|T|$ and $T$ is infinite. Then there exists an enumeration $S=\left\{s_{t}: t \in T\right\}$ such that $\left|\left\{t \in T: s_{t}=s\right\}\right|=|T|$ for every $s \in S$.

Proof. Since $1 \leq|S| \leq|T|$, we can fix a surjection $f: T \rightarrow S$. Since $T$ is infinite, we have $|T|=|T \times T|$, so we can fix a bijection $\theta: T \rightarrow T \times T$. Let $\pi: T \times T \rightarrow T$ be the projection on the first coordinate. Define $s_{t}=f \circ \pi \circ \theta(t)$ for every $t \in T$. We claim that $\left\{s_{t}: t \in T\right\}$ is the desired enumeration. Indeed, let $s \in S$ be arbitrary. Since $f$ is a surjection, $s=f\left(t_{0}\right)$ for some $t_{0} \in T$. Since $\left|\left\{t_{0}\right\} \times T\right|=|T|$ and $\theta$ is a bijection, the set $T^{\prime}=\theta^{-1}\left(\left\{t_{0}\right\} \times T\right) \subseteq T$ satisfies $\left|T^{\prime}\right|=|T|$. Finally, for every $t \in T^{\prime}$, we have $\pi \circ \theta(t) \in \pi\left(\theta\left(T^{\prime}\right)\right) \in \pi\left(\theta\left(\theta^{-1}\left(\left\{t_{0}\right\} \times T\right)\right)\right)=\pi\left(\left\{t_{0}\right\} \times T\right)=\left\{t_{0}\right\}$, so $s_{t}=f \circ \pi \circ \theta(t)=f\left(t_{0}\right)=s$.

Fix a cardinal $\kappa$ such that $\kappa^{\omega}=\kappa$ and a set $K$ such that $|K|=\kappa$. Let $K=K_{0} \cup K_{1}$ be a partition of $K$ into pairwise disjoint sets $K_{i}$ such that $\left|K_{i}\right|=\kappa$ for $i=0,1$.

Let $P$ be a non-empty subset of $\beta \mathbb{N} \backslash \mathbb{N}$ satisfying $|P| \leq \kappa$. Consider the set

$$
X=P \times K \times(\omega+1)
$$

as in Definition 7. Note that $|X|=\kappa$ by Equation (14) and our assumption on $K, P$, and $\kappa$.

For a set $S$, we denote by $[S] \leq \omega$ the family of at most countable subsets of $S$ and by $[S]^{\omega}$ the family of all countably infinite subsets of $X$. 
Claim 4. (i) There exists an enumeration $[B(X)]^{\omega}=\left\{A_{\beta}: \beta \in K_{0}\right\}$ such that $\left|\left\{\beta \in K_{0}: A_{\beta}=A\right\}\right|=\kappa$ for every $A \in[B(X)]^{\omega}$.

(ii) There exists an enumeration $[P] \leq \omega \times[K] \leq \omega \times B(X)=\left\{\left(P_{\beta}, K_{\beta}, h_{\beta}\right): \beta \in K_{1}\right\}$ such that $\mid\left\{\beta \in K_{1}\right.$ : $\left.P_{\beta}=P^{\prime}, K_{\beta}=K^{\prime}, h_{\beta}=h\right\} \mid=\kappa$ whenever $P^{\prime} \in[P] \leq \omega, K^{\prime} \in[K] \leq \omega$ and $h \in B(X)$.

Proof. (i) Note that $|B(X)|=\left|X^{<\omega}\right|=|X|=\kappa$ and $\left|[B(X)]^{\omega}\right|=\kappa^{\omega}=\kappa=\left|K_{0}\right|$ by our assumption on $\kappa$ and $K_{0}$, so we can apply Lemma 16 (with $S=[B(X)]^{\omega}$ and $T=K_{0}$ ) to fix the desired enumeration $[B(X)]^{\omega}=\left\{A_{\beta}: \beta \in K_{0}\right\}$.

(ii) Since $|P| \leq \kappa$ and $|K|=|B(X)|=\kappa$, we have $|[P] \leq \omega \times[K] \leq \omega \times B(X)| \leq \kappa^{\omega}=\kappa=\left|K_{1}\right|$, so the existence of the desired enumeration $[P] \leq \omega \times[K] \leq \omega \times B(X)=\left\{\left(P_{\beta}, K_{\beta}, h_{\beta}\right): \beta \in K_{1}\right\}$ follows from Lemma 16 applied with $S=[P] \leq \omega \times[K] \leq \omega \times B(X)$ and $T=K_{1}$.

For every $\beta \in K$, we define a coherent map $f_{\beta}: X \rightarrow \mathbb{Z}_{2}$ differently depending on whether $\beta \in K_{0}$ or $\beta \in K_{1}$.

Case 1. $\beta \in K_{0}$. In this case, we use a Theorem 2 to fix a coherent map $f_{\beta}: X \rightarrow \mathbb{Z}_{2}$ splitting $A_{\beta}$.

Case 2. $\beta \in K_{1}$. If $h_{\beta} \in B(X) \backslash B\left(P_{\beta} \times K_{\beta} \times(\omega+1)\right)$, then we use Lemma 6 to fix a coherent map $f_{\beta}: X \rightarrow \mathbb{Z}_{2}$ such that $\tilde{f}_{\beta}\left(B\left(P_{\beta} \times K_{\beta} \times(\omega+1)\right)\right) \subseteq\{0\}$ and $\tilde{f}_{\beta}\left(h_{\beta}\right)=1$; otherwise, we let $f_{\beta}$ to be the constant map sending $X$ to $\{0\}$ (this map is clearly coherent).

Claim 5. There exist an enumeration $[K]^{\omega}=\left\{I_{k}: k \in K\right\}$ and a sequence $\left\{y_{k, n}: n \in \omega\right\} \subseteq \mathbb{Z}_{2}^{I_{k}}$ for every $k \in K$ such that whenever $I \in[K]^{\omega}$ and $\left\{y_{n}: n \in \omega\right\} \subseteq \mathbb{Z}_{2}^{I}$, one can find $k \in K$ with $I_{k}=I$ and $y_{k, n}=y_{n}$ for all $n \in \mathbb{N}$.

Proof. Let $S=\bigcup\left\{\left(\mathbb{Z}_{2}^{I}\right)^{\omega}: I \in[K]^{\omega}\right\}$. (We recall that $\left(\mathbb{Z}_{2}^{I}\right)^{\omega}$ denotes the set of all functions from $\omega$ to $\mathbb{Z}_{2}^{I}$; each such function $s$ can be considered as a sequence $\{s(n): n \in \omega\}$ of points of $\mathbb{Z}_{2}^{I}$.)

Since $\left|\left(\mathbb{Z}_{2}^{I}\right)^{\omega}\right|=\mathfrak{c} \leq \kappa$ for every $I \in[K]^{\omega}$, we have $|S| \leq \kappa^{\omega}=\kappa=|K|$ by our assumption on $\kappa$. Therefore, we can apply Lemma 16 with $T=K$ to fix an enumeration $S=\left\{s_{k}: k \in K\right\}$ such that $\left\{k \in K: s_{k}=s\right\}$ has cardinality $\kappa$ for every $s \in S$.

Let $k \in K$. Then $s_{k} \in S$, so $s_{k} \in\left(\mathbb{Z}_{2}^{I}\right)^{\omega}$ for a unique $I \in[K]^{\omega}$; that is, $s_{k}$ is a function from $\omega$ to $\mathbb{Z}_{2}^{I}$. We define $I_{k}=I$ and $y_{k, n}=s_{k}(n)$ for all $n \in \omega$.

Let $I \in[K]^{\omega}$ and $\left\{y_{n}: n \in \omega\right\} \subseteq \mathbb{Z}_{2}^{I}$ be arbitrary. Then the function $s: \omega \rightarrow \mathbb{Z}_{2}^{I}$, defined by $s(n)=$ $y_{n}$ for $n \in \omega$, belongs to $S$. By the choice of our enumeration, the set $\left\{k \in K: s_{k}=s\right\}$ has cardinality $\kappa$. In particular, there exists $k \in K$ such that $s=s_{k}$. Now $I_{k}=I$ and $y_{n}=s(n)=s_{k}(n)=y_{k, n}$ for every $n \in \omega$.

Define

$$
y_{p, k, n}=y_{k, n} \text { for all }(p, k, n) \in P \times K \times \omega .
$$

For each $(p, k) \in P \times K$, the sequence $\left\{y_{p, k, n}: n \in \omega\right\}=\left\{y_{k, n}: n \in \omega\right\}$ of points of the compact space $\mathbb{Z}_{2}^{I_{k}}$ has a $p$-limit $y_{p, k, \omega} \in \mathbb{Z}_{2}^{I_{k}}$.

For each $(p, k, n) \in X$, define $z_{p, k, n} \in \mathbb{Z}_{2}^{K}$ by

$$
z_{p, k, n}(\beta)=\left\{\begin{array}{ll}
y_{p, k, n}(\beta) & \text { if } \beta \in I_{k} \\
f_{\beta}(p, k, n) & \text { if } \beta \in K \backslash I_{k}
\end{array} \quad \text { for every } \beta \in K\right.
$$

Claim 6. For every $p \in P$ and each sequence $\left\{W_{n}: n \in \mathbb{N}\right\}$ of non-empty open subsets of $\mathbb{Z}_{2}^{K}$, there exists $k \in K$ such that

(i) $z_{p, k, n} \in W_{n}$ for all $n \in \mathbb{N}$, and

(ii) $z_{p, k, \omega}$ is a $p$-limit of the sequence $\left\{z_{p, k, n}: n \in \mathbb{N}\right\}$. 
Proof. Fix $p \in P$ and a sequence $\left\{W_{n}: n \in \mathbb{N}\right\}$ of non-empty open subsets of $\mathbb{Z}_{2}^{K}$. Without loss of generality, we may assume that each $W_{n}$ is a basic open subset of $\mathbb{Z}_{2}^{K}$; that is, $W_{n}=\prod_{\beta \in K} W_{\beta, n}$, where each $W_{\beta, n}$ is a non-empty (open) subset of $\mathbb{Z}_{2}$ and $\operatorname{supp}\left(W_{n}\right)=\left\{\beta \in K: W_{\beta, n} \neq \mathbb{Z}_{2}\right\}$ is a finite subset of $K$. Then the set $J=\bigcup_{n \in \mathbb{N}} \operatorname{supp}\left(W_{n}\right)$ is at most countable, so we can fix a countably infinite subset $I$ of $K$ containing $J$. For every $n \in \mathbb{N}, V_{n}=\prod_{\beta \in I} W_{\beta, n}$ is a non-empty subset of $\mathbb{Z}_{2}^{I}$, so we can select $y_{n} \in V_{n}$. By Equation (15) and Claim 5, there exists $k \in K$ such that $I_{k}=I$ and $y_{p, k, n}=y_{k, n}=y_{n}$ for all $n \in \mathbb{N}$.

(i) Fix $n \in \mathbb{N}$. By Equation (16), we have

$$
z_{p, k, n}(\beta)=y_{p, k, n}(\beta)=y_{n}(\beta) \in W_{\beta, n} \text { for every } \beta \in I_{k}=I .
$$

Since $\operatorname{supp}\left(W_{n}\right) \subseteq I$, this implies $z_{p, k, n} \in W_{n}$.

(ii) It suffices to check that $z_{p, k, \omega}(\beta)$ is a $p$-limit of the sequence $\left\{z_{p, k, n}(\beta): n \in \mathbb{N}\right\}$ for every $\beta \in K$. We consider two cases.

Case 1. $\beta \in I_{k}$. Since the sequence $\left\{y_{p, k, n}: n \in \mathbb{N}\right\} \subseteq \mathbb{Z}_{2}^{I_{k}}$ has a $p$-limit $y_{p, k, \omega} \in \mathbb{Z}_{2}^{I_{k}}$, it follows that $y_{p, k, \omega}(\beta)$ is a $p$-limit of the sequence $\left\{y_{p, k, n}(\beta): n \in \mathbb{N}\right\}$. Since $\beta \in I_{k}$, we have $z_{p, k, \omega}(\beta)=y_{p, k, \omega}(\beta)$ by Equation (16). Combining this with Equation (17), we obtain the desired conclusion.

Case 2. $\beta \in K \backslash I_{k}$. In this case, it follows from Equation (16) that $z_{p, k, n}(\beta)=f_{\beta}(p, k, n)$ for every $n \in \omega+1$, and the conclusion follows from the fact that $f_{\beta}$ is coherent.

Claim 7. The set

$$
Z=\left\{z_{p, k, n}:(p, k, n) \in X\right\}
$$

is dense in $\mathbb{Z}_{2}^{K}$.

Proof. Consider an arbitrary non-empty open subset $U$ of $\mathbb{Z}_{2}^{K}$. Let $W_{n}=U$ for every $n \in \mathbb{N}$. Since $P$ is non-empty, we can choose $p \in P$. Let $k \in K$ be as in the conclusion of Claim 6 applied to this $p$ and the sequence $\left\{W_{n}: n \in \mathbb{N}\right\}$. Then $z_{p, k, 1} \in W_{1}=U$. Since $(p, k, 1) \in X$ by Equation (14), we obtain $z_{p, k, 1} \in Z$ by Equation (18), so $Z \cap U \neq \varnothing$.

Claim 8. $Z$ is strongly P-pseudocompact.

Proof. By Definition 4 (ii), we need to check that $Z$ is strongly $p$-pseudocompact for every $p \in P$. Fix $p \in P$. Let $\left\{U_{n}: n \in \mathbb{N}\right\}$ be a sequence of non-empty open subsets of $Z$. Since $Z$ is a subspace of $\mathbb{Z}_{2}^{K}$, for every $n \in \mathbb{N}$, there exists an open subset $W_{n}$ of $Z_{2}^{K}$ such that $U_{n}=Z \cap W_{n}$; in particular, $W_{n}$ is non-empty. Let $k \in K$ be as in the conclusion of Claim 6 applied to $p$ and the sequence $\left\{W_{n}: n \in \mathbb{N}\right\}$. By Item (i) of this claim, we have $z_{p, k, n} \in W_{n}$ for every $n \in \mathbb{N}$. Since $(p, k, n) \in X$ by Equation (14), $z_{p, k, n} \in Z$ by Equation (18), so $z_{p, k, n} \in Z \cap W_{n}=U_{n}$ for every $n \in \mathbb{N}$. By Item (ii) of Claim $6, z_{p, k, \omega}$ is a $p$-limit of the sequence $\left\{z_{p, k, n}: n \in \mathbb{N}\right\}$. Since $(p, k, \omega) \in X$ by Equation (14), we obtain $z_{p, k, \omega} \in Z$ by Equation (18). According to Definition 1, this shows that $Z$ is strongly $p$-pseudocompact.

Let $G$ be the subgroup of $\mathbb{Z}_{2}^{K}$ generated by $Z$. Let $f: X \rightarrow Z \subseteq G$ be the map defined by

$$
f(p, k, n)=z_{p, k, n} \text { for every }(p, k, n) \in X
$$

Since $G$ is a Boolean group, there exists a unique homomorphism $\tilde{f}: B(X) \rightarrow G$ extending $f$. Since $f(X)=Z$ and the latter set algebraically generates $G$, the homomorphism $\tilde{f}$ is surjective.

Claim 9. For every at most countable set $A \subseteq B(X)$, there exists an at most countable set $I \subseteq K$ such that

$$
\pi_{\beta} \circ \tilde{f}(a)=\tilde{f}_{\beta}(a) \text { whenever } \beta \in K \backslash I \text { and } a \in A,
$$


where $\pi_{\beta}: \mathbb{Z}_{2}^{K} \rightarrow \mathbb{Z}_{2}$ is the projection on $\beta^{\prime}$ th coordinate.

Proof. For every $a \in A$, there exists a finite set $E_{a} \subseteq X$ such that

$$
a=\sum_{(p, k, n) \in E_{a}}\{(p, k, n)\} .
$$

Since $A$ is at most countable, so is the set

$$
J=\left\{k \in K: \exists p \in P \exists n \in(\omega+1)(p, k, n) \in \bigcup\left\{E_{a}: a \in A\right\}\right\} .
$$

Therefore, $I=\bigcup_{k \in J} I_{k}$ is an at most countable subset of $K$.

Let $a \in A$ and $\beta \in K \backslash I$ be arbitrary. Suppose that $(p, k, n) \in E_{a}$. Then $k \in J$ by Equation (22). Therefore, $I_{k} \subseteq I$ by our choice of $I$. Since $\beta \notin I$, we conclude that $\beta \in K \backslash I_{k}$; thus, $z_{p, k, n}(\beta)=f_{\beta}(p, k, n)$ by Equation (16). Since this holds for every $(p, k, n) \in E_{a}$ and $\tilde{f}_{\beta}$ is a homomorphism, from Equations (19) and (21) we conclude that

$$
\begin{gathered}
\tilde{f}_{\beta}(a)=\tilde{f}_{\beta}\left(\sum_{(p, k, n) \in E_{a}}\{(p, k, n)\}\right)=\sum_{(p, k, n) \in E_{a}} \tilde{f}_{\beta}(\{(p, k, n)\})=\sum_{(p, k, n) \in E_{a}} f_{\beta}(p, k, n)=\sum_{(p, k, n) \in E_{a}} z_{p, k, n}(\beta) \\
=\sum_{(p, k, n) \in E_{a}} f(p, k, n)(\beta)=\tilde{f}\left(\sum_{(p, k, n) \in E_{a}}\{(p, k, n)\}\right)(\beta)=\tilde{f}(a)(\beta)=\pi_{\beta} \circ \tilde{f}(a) .
\end{gathered}
$$

This proves Equation (20).

Claim 10. G contains no non-trivial convergent sequences.

Proof. Consider an arbitrary countably infinite set $S \subseteq G$. Since $\tilde{f}: B(X) \rightarrow G$ is a surjection, we can fix a countably infinite set $A \subseteq B(X)$ such that $\tilde{f}(A)=S$ and $\tilde{f} \uparrow_{A}: A \rightarrow S$ is a bijection. Let $I \subseteq K$ be the set as in the conclusion of Claim 9 (applied to our $A$ ). Since $A \in[B(X)]^{\omega}$, we can apply Claim 4 (i) to conclude that the set $\left|\left\{\beta \in K_{0}: A_{\beta}=A\right\}\right|$ has cardinality $\kappa$. Since $\left|K_{0}\right|=\kappa \geq \mathfrak{c}>\omega \geq|I|$, there exists $\beta \in K_{0} \backslash I$. Then $f_{\beta}$ splits the set $A=A_{\beta}$ by our choice of $f_{\beta}$. This means that the set $A_{i}=\left\{a \in A: \tilde{f}_{\beta}(a)=i\right\}$ is infinite for both $i \in \mathbb{Z}_{2}$.

Let $i \in \mathbb{Z}_{2}$ be arbitrary. Since $\tilde{f} \uparrow_{A}: A \rightarrow S$ is a bijection, the set $S_{i}=\tilde{f}\left(A_{i}\right) \subseteq S$ is infinite. It follows from Equation (20) that $\pi_{\beta} \circ \tilde{f}(a)=\tilde{f}_{\beta}(a)=i$ for $a \in A_{i}$, so $\pi_{\beta}(s)=i$ for $s \in S_{i}$. Since the map $\pi_{\beta}$ is continuous, it follows that $S_{i}$ is a closed subset of $S$.

Since $\tilde{f} \uparrow_{A}: A \rightarrow S$ is a bijection and $A=A_{0} \cup A_{1}$ is a partition of $A$ into disjoint sets $A_{i}$, it follows that $S=S_{0} \cup S_{1}$ is a partition of $S$ into disjoint sets $S_{i}$. Since each $S_{i}$ is infinite and closed in $S$, this implies that $S$ cannot be a convergent sequence in $G$.

Claim 11. If $P^{\prime} \in[P] \leq \omega$ and $K^{\prime} \in[K] \leq \omega$, then the subgroup $H_{P^{\prime}, K^{\prime}}$ of $G$ generated by the set

$$
Z_{P^{\prime}, K^{\prime}}=\left\{z_{p, k, n}: p \in P^{\prime}, k \in K^{\prime}, n \in \omega+1\right\}
$$

is closed in $G$.

Proof. Fix $P^{\prime} \in[P] \leq \omega$ and $K^{\prime} \in[K]^{\leq \omega}$. Note that $\tilde{f}\left(B\left(P^{\prime} \times K^{\prime} \times(\omega+1)\right)\right)=H_{P^{\prime}, K^{\prime}}$ by Equations (19) and (23).

Let $g \in G \backslash H_{P^{\prime}, K^{\prime}}$ be arbitrary. Since $\tilde{f}$ is surjective, $\tilde{f}(h)=g$ for some $h \in B(X)$. Clearly, $h \notin B\left(P^{\prime} \times K^{\prime} \times(\omega+1)\right)$. Apply Claim 9 to at most countable subset

$$
A=B\left(P^{\prime} \times K^{\prime} \times(\omega+1)\right) \cup\{h\}
$$


of $B(X)$ to obtain at most countable set $I \subseteq K$ as in the conclusion of this claim. Since $\left(P^{\prime}, K^{\prime}, h\right) \in$ $[P] \leq \omega \times[K] \leq \omega \times B(X)$, we can apply Claim 4 (ii) to conclude that the set $K_{1}^{\prime}=\left\{\beta \in K_{1}: P_{\beta}=P^{\prime}, K_{\beta}=\right.$ $\left.K^{\prime}, h_{\beta}=h\right\}$ has cardinality $\kappa$. Since $\left|K_{1}^{\prime}\right|=\kappa \geq \mathfrak{c}>\omega \geq|I|$, there exists $\beta \in K_{1}^{\prime} \backslash I$. Then $P_{\beta}=P^{\prime}, K_{\beta}=$ $K^{\prime}$ and $h_{\beta}=h$. Since $h_{\beta}=h \in B(X) \backslash B\left(P^{\prime} \times K^{\prime} \times(\omega+1)\right)=B(X) \backslash B\left(P_{\beta} \times K_{\beta} \times(\omega+1)\right)$ by our assumption, it follows from $\beta \in K_{1}^{\prime} \subseteq K_{1}$ and our choice of $f_{\beta}$ that $\tilde{f}_{\beta}\left(B\left(P^{\prime} \times K^{\prime} \times(\omega+1)\right)\right) \subseteq\{0\}$ and $\tilde{f}_{\beta}(h)=1$. From this, $\beta \in K \backslash I$, Equations (20) and (24), we conclude that $\pi_{\beta} \circ \tilde{f}\left(B\left(P^{\prime} \times K^{\prime} \times\right.\right.$ $(\omega+1))) \subseteq\{0\}$ and $\pi_{\beta} \circ \tilde{f}(h)=1$. Since $H_{P^{\prime}, K^{\prime}}=\tilde{f}\left(B\left(P^{\prime} \times K^{\prime} \times(\omega+1)\right)\right)$ and $g=\tilde{f}(h)$, we get $\pi_{\beta}\left(H_{P^{\prime}, K^{\prime}}\right) \subseteq\{0\}$ and $\pi_{\beta}(g)=1$. Since $\pi_{\beta}$ is continuous, $U_{g}=\pi_{\beta}^{-1}(1)$ is an open neighbourhood of $g$ in $G$ disjoint from $H_{P^{\prime}, K^{\prime}}$.

For every $g \in G \backslash H_{P^{\prime}, K^{\prime}}$, we found an open neighbourhood $U_{g}$ of $G$ such that $U_{g} \cap H_{P^{\prime}, K^{\prime}}=\varnothing$. Therefore, $H_{P^{\prime}, K^{\prime}}$ is closed in $G$.

Claim 12. The closure of each at most countable subset of $G$ is at most countable.

Proof. Let $S$ be an at most countable subset of $G$. Since $Z$ algebraically generates $G$, from Equations (18) and (23) we conclude that there exist $P^{\prime} \in[P] \leq \omega$ and $K^{\prime} \in[K] \leq \omega$ such that $S \subseteq H_{P^{\prime}, K^{\prime}}$. (Recall that $H_{P^{\prime}, K^{\prime}}$ is algebraically generated by $Z_{P^{\prime}, K^{\prime}}$.) Since $H_{P^{\prime}, K^{\prime}}$ is closed in $G$ by Claim 11 , the closure of $S$ is contained in $H_{P^{\prime}, K^{\prime}}$. Since $P^{\prime}$ and $K^{\prime}$ are at most countable, so is $Z_{P^{\prime}, K^{\prime}}$ and thus $H_{P^{\prime}, K^{\prime}}$ as well.

Claim 13. All separable pseudocompact subsets of $G$ are finite.

Proof. This follows from Claims 10 and 12 and Lemma 10.

Since $Z \subseteq G \subseteq \mathbb{Z}_{2}^{K}$, and $Z$ is dense in $\mathbb{Z}_{2}^{K}$ by Claim $7, Z$ is dense in $G$. Since $Z$ is strongly $P$-pseudocompact by Claim 8 , so is $G$. By Claim 13, G does not contain infinite separable pseudocompact subsets. Finally, since $|K|=\kappa$, the topological groups $\mathbb{Z}_{2}^{K}$ and $\mathbb{Z}_{2}^{\kappa}$ are topologically isomorphic.

\section{Further Open Questions}

In this section we list natural open questions (besides Question 2) inspired by our results.

As was mentioned in Section 8, Galindo and Macario proved that, under a mild additional set-theoretic assumption beyond ZFC, every pseudocompact abelian group admits a pseudocompact group topology without infinite compact subsets [12]. Question 4 below asks for an analogue of their result for other compactness-like properties listed on the left side of Figure 1, while Question 3 is a version of Question 4 restricted to non-trivial convergent sequences. Item (iv) was excluded in Question 4 due to Remark 1 (ii).

Question 3. Let $\mathcal{P}$ be one of the following properties:

(i) selectively pseudocompact;

(ii) strongly p-pseudocompact for some $p \in \beta \mathbb{N} \backslash \mathbb{N}$;

(iii) strongly $(\beta \mathbb{N} \backslash \mathbb{N})$-pseudocompact;

(iv) strongly $(\beta \mathbb{N} \backslash \mathbb{N})$-bounded.

If an infinite abelian group admits a group topology with property $\mathcal{P}$, must it also admit a group topology with property $\mathcal{P}$ having no non-trivial convergent sequences?

Question 4. Let $\mathcal{P}$ be one of the properties (i)-(iii) from Question 3. If an infinite abelian group admits a group topology with property $\mathcal{P}$, must it also admit a group topology with property $\mathcal{P}$ having no infinite compact subsets (or even without infinite separable pseudocompact subsets)? 
It makes no sense to ask Questions 3 and 4 for properties on the right side of Figure 1, because infinite selectively sequentially pseudocompact spaces contain non-trivial convergent sequences ([5], Proposition 3.1).

Question 5. If an abelian group admits a pseudocompact group topology, must it also admit a group topology having one of the stronger properties (i)-(iv) listed in Question 3?

The version of Question 5 for "selective pseudocompactness" is due to García-Ferreira and Tomita ([7], Question 2.7).

Our last question is related to the reversibility of Arrow 4 in Figure 1 in the class of topological groups.

Question 6. Does there exist a ZFC example of a selectively pseudocompact (abelian) group which is not strongly p-pseudocompact for any free ultrafilter $p$ on $\mathbb{N}$ ?

An example under $\mathrm{CH}$ is mentioned in the text after Figure 1.

Author Contributions: Both authors contributed equally to this research work.

Funding: The first listed author was partially supported by the Grant-in-Aid for Scientific Research (C) No. JP26400091 of the Japan Society for the Promotion of Science (JSPS). The second listed author was partially supported by the 2016/2017 fiscal year grant of the Matsuyama Saibikai.

Conflicts of Interest: The authors declare no conflict of interest.

\section{References}

1. Bernstein, A.R. A new kind of compactness for topological spaces. Fund. Math. 1970, 66, 185-193. [CrossRef]

2. Angoa, J.; Ortiz-Castillo, Y.F; Tamariz-Mascarúa, A. Compact-like properties in hyperspaces. Matematički Vesnik 2013, 65, 306-318.

3. Angoa, J.; Ortiz-Castillo, Y.F.; Tamariz-Mascarúa, A. Ultrafilters and properties related to compactness. Topol. Proc. 2014, 43, 183-200.

4. García-Ferreira, S.; Ortiz-Castillo, Y.F. Strong pseudocompact properties. Comment. Math. Univ. Carol. 2014, 55, 101-109.

5. Dorantes-Aldama, A.; Shakhmatov, D. Selective sequential pseudocompactness. Topol. Appl. 2017, 222, 53-69. [CrossRef]

6. García-Ferreira, S.; Tomita, A.H. Finite powers of selectively pseudocompact groups. Topol. Appl. 2018, 248, 50-58. [CrossRef]

7. García-Ferreira, S.; Tomita, A.H. A pseudocompact group which is not strongly pseudocompact. Topol. Appl. 2015, 192, 138-144. [CrossRef]

8. Shakhmatov, D.; Yañez, V. The impact of the Bohr topology on selective pseudocompactness. arXiv 2018, arXiv:1801.09380.

9. Hernández, S.; Macario, S. Dual properties in totally bounded Abelian groups. Arch. Math. 2003, 80, 271-283. [CrossRef]

10. Arhangel'skiı̌, A.V. Topological Function Spaces; Kluwer: Dordrecht, The Netherlands, 1991; Volume 78.

11. Ardanza-Trevijano, S.; Chasco, M.J.; Domínguez, X.; Tkachenko, M. Precompact noncompact reflexive Abelian groups. Forum Math. 2012, 24, 289-302. [CrossRef]

12. Galindo, J.; Macario, S. Pseudocompact group topologies with no infinite compact subsets. J. Pure Appl. Algebra 2011, 215, 655-663. [CrossRef]

13. Malykhin, V.I.; Shapiro, L.B. Pseudocompact groups without convergent sequences. Math. Notes Acad. Sci. USSR 1985, 37, 59-62. (In Russian)

14. Dijkstra, J.J.; van Mill, J. Groups without convergent sequences. Topol. Appl. 1996, 74, 275-282. [CrossRef]

15. Dikranjan, D.; Shakhmatov, D. Algebraic structure of pseudocompact groups. Mem. Am. Math. Soc. 1998, 133, 633. [CrossRef]

16. Higman, G. Unrestricted free products, and varieties of topological groups. J. Lond. Math. Soc. 1952, 27, 73-81. [CrossRef] 
17. Morris, S.A. Varieties of topological groups. Bull. Aust. Math. Soc. 1969, 1, 145-160. [CrossRef]

18. Comfort, W.W.; van Mill, J. On the existence of free topological groups. Topol. Appl. 1988, 29, $245-269$. [CrossRef]

19. Morris, S.A. Varieties of topological groups: A survey. Colloq. Math. 1982, 46, 147-165. [CrossRef]

20. Shakhmatov, D.; Spěvak, J. Group valued continuous functions with the topology of pointwise convergence. Topol. Appl. 2010, 157, 1518-1540. [CrossRef]

21. Shakhmatov, D. Imbeddings into topological groups preserving dimensions. Topol. Appl. 1990, 36, 181-204. [CrossRef]

22. Sipacheva, O. Free Boolean topological groups. Axioms 2015, 4, 492-517. [CrossRef]

23. Tkachenko, M.G.; Yaschenko, I. Independent group topologies on Abelian groups. Topol. Appl. 2002, 122, 425-451. [CrossRef]

24. Arhangel'skiı̌, A.V. An addition theorem for the weight of sets lying in bicompacts. Dokl. Akad. Nauk SSSR 1959, 126, 239-241. (In Russian)

25. Galindo, J.; García-Ferreira, S. Compact groups containing dense pseudocompact subgroups without non-trivial convergent sequences. Topol. Appl. 2007, 154, 476-490. [CrossRef]

26. Sirota, S.M. The product of topological groups, and extremal disconnectedness. Sb. Math. 1969, 8, 169-180. (In Russian) [CrossRef]

27. Chasco, M.J.; Dikranjan, D.; Martín-Peinador, E. A survey on reflexivity of abelian topological groups. Topol. Appl. 2012, 159, 2290-2309. [CrossRef]

28. Glicksberg, I. Uniform boundedness for groups. Can. J. Math. 1962, 14, 269-276. [CrossRef]

29. Dikranjan, D.; Shakhmatov, D. A Kronecker-Weyl theorem for subsets of abelian groups. Adv. Math. 2011, 226, 4776-4795. [CrossRef]

30. Dikranjan, D.; Shakhmatov, D. Hewitt-Marczewski-Pondiczery type theorem for abelian groups and Markov's potential density. Proc. Am. Math. Soc. 2010, 138, 2979-2990. [CrossRef]

(C) 2018 by the authors. Licensee MDPI, Basel, Switzerland. This article is an open access article distributed under the terms and conditions of the Creative Commons Attribution (CC BY) license (http://creativecommons.org/licenses/by/4.0/). 